\title{
Thermally-driven flows in polymeric liquids
}

\author{
Marcello Lappa \\ Department of Mechanical and Aerospace Engineering, University of Strathclyde \\ James Weir Building, 75 Montrose Street, Glasgow, G1 1XJ, UK \\ email: marcello.lappa@strath.ac.uk
}

\begin{abstract}
Within the vast array of problems related to the processing of plastic materials and polymeric liquids, the main focus of the present chapter is the illustration of the typical dynamics of thermally-driven flows in these fluids, i.e. 'natural' fluid motion induced by gravity or surfacetension effects. Experimental struggles with understanding the basic mechanisms of these fundamental types of convection in viscoelastic fluids have been mitigated over recent years by the development of relevant models and adequate numerical techniques. On the one hand, this progress has produced new fundamental knowledge; on the other hand, it has led to new questions, both general and system-specific (a relevant example being represented by the intriguing concept of "elastic turbulence"). This field continues to burgeon and bring surprises to this day. A synthetic review of the salient outcomes of past and recent studies is reported in this chapter (articulated in focused subsections) together with a critical discussion of some accompanying necessary definitions and paradigms used to reveal the intimate essence of these liquids. For the convenience of the reader, however, advanced formal discussions and mathematical arguments are stripped down to their essentials. In the same spirit, the use of jargon is kept at minimum and much of the available space is devoted to the description (both qualitative and quantitative) of the considered convective phenomena, the related thresholds in terms of characteristic numbers and the underlying "physics".
\end{abstract}

Keywords: Viscoelastic fluids, Buoyancy convection, surface-tension driven convection, elastic turbulence.

\section{Introduction}

Plastic materials are central in several technological applications in the chemical, materials, mechanical, automotive, aerospace, energy and food industries. Often related processing techniques involve one or more stages in which extended portions of these materials pass through a (partially or completely) molten state. Relevant examples are plastics joining (Rotheiser, 1999; Troughton, 2008), heating of polymers required for mechanical and tribological properties improvement (Aly, 2015 and references therein), the welding of plastics (Grewell and Benatar, 2007), fiber spinning and fiber casting, film blowing and extrusion processes (Dawson, 1999; Chan and Chung, 2010; Rauwendaal, 2013; Arshad et al., 2014), and many other applications in engineering where processing of thermoplastics involves a polymer melt (Bonito et al., 2011).

Obviously, all these applications implicitly involve the existence of thermal gradients across a phase boundary or through a spatially extended region of molten material. The components in various directions of these gradients can produce 'natural' (we will specify the precise meaning of this word later) fluid motion that can interfere with the abovementioned processes in several ways, e.g., by leading to significant alterations in the intended distribution of temperature, causing a strong 
increase in the ability of these materials to transport heat, and/or inducing fluid mixing and even turbulence in some specific circumstances.

Molten plastics and related polymeric liquids are therefore often subjected to out-of-control heatand mass-transfer conditions, which can often cause undesired effects and morphological alterations or defects in the final products (Hitt and Smith, 1993; Guto et al., 1995). In practice, the essentially unpredictable behavior of these flows essentially follows from their physical origin, which as explained above, is 'natural', i.e. it is a direct consequence of mechanisms induced by body or surface forces present in the liquid.

Body forces are essentially represented by gravity, which is obviously omnipresent in all technological processes. Surface forces start to play a role when the considered liquid is directly in contact with a gas (e.g., air). The existence of a boundary separating the liquid from a gaseous phase (i.e. an 'interface') implies surface tension, that is, an inward force resulting from an imbalance between the mutual attraction among liquid molecules (relatively strong) and than that existing between the liquid and gas molecules (weak).

In non-isothermal conditions, these forces can produce fluid motion. The related cause-and-effect relationship is relatively obvious in the case of gravity. The density of liquids generally behaves as a decreasing function of temperature. This means that the density of the liquid is relatively higher in regions where the temperature is smaller and vice versa it undergoes a decrease where the liquid is warmer. In the presence of a temperature difference, hot (lighter) fluid therefore tends to rise while cold (heavier) fluid sinks. This process typically leads to a pervasive (involving the entire bulk) fluid motion, generally known as buoyancy or 'thermogravitational convection'.

Though the explanation for surface-tension driven convection is less straightforward, it is based on similar principles. Like density, also the surface tension of most of known liquids behaves as a decreasing function of temperature. If a temperature gradient is established along an interface, an imbalance is created in the corresponding distribution of surface tension. As a result, fluid tends to move from the region of the liquid-gas interface where the surface tension is smaller (hot side) towards the region where it is higher (cold side). Due to viscosity, surface motion is propagated to more internal regions, where it can even reverse its sense of motions due to 'continuity' (mass must be conserved). The final effect is therefore a circulation where the fluid moves from the hot side to the cold one along the interface and in the opposite direction at a certain distance from the free interface (the so-called 'return' flow).

Remarkably, convection of this type is not an exclusive prerogative of situations where the temperature changes along the interface. It can even emerge from quiescent fluids for which the temperature of the interface is perfectly uniform (no gradient), provided a temperature difference is established along a direction perpendicular to the free surface.

In order to clarify these dynamics, it is therefore necessary to provide a rigorous classification of all these forms of convection on the basis of the dominant physical force and the prevailing direction where temperature changes occur. 
For thermogravitational convection, in particular, two archetypal situations are possible. Temperature difference $(\Delta T)$ parallel or perpendicular to gravity. The first case is known as the Rayleigh-Bénard problem (RB) and corresponds to the paradigm of a fluid uniformly heated from below and cooled from above. The second situation is typically attained when a fluid encapsulated in a rectangular container is heated at one side and cooled at the other side (generally known as the Hadley convection problem).

A similar classification holds for the case of surface-tension-induced convection. If the prevailing temperature gradient is perpendicular to the free interface (e.g., the fluid layer is heated from below and exchanges heat with the external environment at the free interface), then the emerging flow is known as Marangoni-Bénard convection (MB). A rotation of the temperature gradient by $90^{\circ}(\Delta T$ parallel to the free surface) turns this type of fluid motion into another convective mechanism simply known as thermocapillary or Marangoni convection.

This articulated nomenclature reflects typical differences relating to the patterning behavior of these convective states and the different evolutionary path that they follow when the temperature difference is progressively increased.

In this regard, the direction of the temperature difference plays a very important role for both gravitational and surface-tension driven flows. Regardless of the force driving the flow (gravity or surface-tension gradient), if the $\Delta T$ is parallel to gravity (RB) or perpendicular to the free surface (MB), convection is produced only if a certain temperature difference is exceeded (critical threshold). If the $\Delta T$ is perpendicular to gravity (Hadley flow) or parallel to the free surface (Marangoni flow), fluid motion develops as soon as some heating is applied (regardless of its magnitude).

In general, for all these cases, on increasing progressively the $\Delta T$ the flow undergoes a series of transitions in terms of flow structure and complexity until a fully turbulent state is attained. Further characterization of these modes of convection, however, is not possible unless the details of the specific fluid under consideration are known or specified.

In this regard, it should be stressed that the knowledge of these phenomena with regard to molten plastics or polymeric liquids is still relatively limited since even modern experimental techniques have a limited ability to visualize convective motion inside these fluids, which in most cases are not transparent to visible light and can display many fine details on several spatial and time scales.

Over recent years, however, methods of analysis based on the numerical solution of the related governing equations (CFD, i.e. Computational Fluid Dynamics) have gained considerable interest as a viable option to the investigation of all these aspects. As with these methods it is possible to unravel processes that are generally interwoven (or overshadowed by other mechanisms in experiments), they are nowadays regarded as powerful tools to deepen the interpretations of available experimental observations and/or to explore parts of the space of parameters, which would otherwise be out of reach.

In other words, we now are in a situation where the field of CFD for polymeric liquids has reached a sort of maturity. As a valid alternative to past strategies based on expensive trial-and-error 
experiments, recent progress in related models and in the availability of adequate resources (computing hardware) has provided powerful platforms that make sophisticated computer-aided modeling and simulation of these phenomena possible. Along these lines, the reader expressly interested in these aspects is strongly encouraged to undertake preliminary readings of the next four sections (Sects. 2-5) and become familiar with the modeling hierarchy described there (fundamental concepts, symbolism, terminology and equations). Vice versa, readers more interested in purely physical or 'phenomenological' details may skip those parts and jump directly into the sections where typical dynamics of these forms of convection are described (Sects. 6-9).

\section{Microscopic interpretation of liquids and related classification}

There are different possible ways to classify a liquid. As an example, liquids can be categorized on the basis of the relative importance of thermal and viscous effects. They can be extremely viscous and have a relatively small thermal diffusivity $(\alpha)$. This is the case for instance of many organic substances such as silicone oils (polymerized siloxane with organic side chains) and many plastic materials in the molten state or polymer solutions. For these fluids the so-called Prandtl number, defined as the ratio of the kinematic viscosity $(v)$ and thermal diffusivity (i.e. $\operatorname{Pr}=v / \alpha$ ) is typically relatively large $(\operatorname{Pr} \geq \mathrm{O}(1))$. Vice versa, fluids also exist for which the ability to conduct heat is much stronger than the ability to transfer momentum. These are the liquid metals (for which typically $v$ is much smaller than $\alpha$, and, therefore, $\operatorname{Pr}<\mathrm{O}(1)$ ). A third independent category of liquids is represented by the molten salts for which $v \cong \alpha$ and $\operatorname{Pr}=O(1)$.

Though this approach to categorize liquids has enjoyed a widespread use in the literature (it can indeed provide some immediate insights into the physical behavior of the considered fluid), however, it is not exhaustive.

Liquids also differ with respect to their ability to develop stresses when subjected to external stimuli. While fluids such as liquid metals, water or other common substances can produce internal stresses only as a result of purely frictional (viscous) effects, molten plastics and polymeric liquids can also develop a second type of stress that does not depend on viscosity and should rather be considered as a consequence of their 'elastic' properties.

A proper mathematical description of these two (elastic and non-elastic) categories of fluids can be elaborated in its simplest form using a statistical mechanics approach. In particular, most conveniently, a distinction can be introduced between liquids consisting of molecules that have compact shape (such as those of water, just to fix ideas) or display an elongated structure such as those typical of plastic materials (macromolecules).

In the former case, fluid particles can be represented as point masses $\left(\mathrm{m}_{\mathrm{i}}\right)$ with velocity $c_{\mathrm{i}}$, obeying

the Newton's law, i.e. $\underline{f}_{i}=m_{i} \frac{d \underline{c}_{i}}{d t}$ (the underlined symbols represent vectors). The generic velocity $\underline{c_{i}}$, in turn, can be split into two contributions: $\underline{c}_{i}=\underline{V}+\underline{C}_{i}$ where $\underline{V}$ is the so-called macroscopic 
fluid velocity while the latter one represents the well-known random motion of molecules (see, e.g., Lappa, 2009).

Using this perspective, the internal stresses emerging in the liquid as a result of the transfer of momentum at microscopic scale among molecules can formally be expressed as

$$
\underline{\underline{\tau}}=-\left\langle\sum_{i=1}^{N}\left[m_{i} \underline{C}_{i} \underline{C}_{i} \delta\left(\underline{r}-\underline{r}_{i}\right)\right]\right\rangle
$$

where $\delta(\underline{r})$ is the Dirac function, $\underline{C}_{\mathrm{i}} \underline{C}_{\mathrm{i}}$ indicates a dyadic product, $\underline{r}_{\mathrm{i}}$ the fluid particle position vector and the symbol $\langle a\rangle$ is used to indicate formally an 'average' value from a stochastic standpoint (practically obtained by integrating the generic quantity $a$ multiplied by a proper distribution function). The next step of this modeling hierarchy relates to the determination of a working link between the stress tensor represented by eq. (1) and the macroscopic liquid velocity $\underline{V}$, needed to allow a description of the macrophysical dynamics of these fluids transcending the microscopic behavior (i.e. with no need to account for the details of the effective distribution of $\underline{C_{\mathrm{i}}}$ ). By invoking some specific assumptions on the nature of the interactions occurring among molecules (binary elastic collisions), the main outcome of this approach (see, e.g., Grad, 1963; Rosenau, 1989) is that the stress tensor can finally be expressed as

$$
\underline{\underline{\tau}}=-p \underline{\underline{I}}+2 \mu(\underline{\nabla} \underline{V})_{o}^{s}
$$

where $p$ is the pressure, $\underline{I}$ is the unit matrix, $(\underline{\nabla} \underline{V})_{o}^{s}$ reads

$$
(\underline{\nabla} \underline{V})^{s}-[(\underline{\nabla} \cdot \underline{V}) \underline{I}] / 3, \text { with }(\underline{\nabla} \underline{V})^{s}=\left(\underline{\nabla} \underline{V}+\underline{\nabla} \underline{V}^{T}\right) / 2
$$

and $\mu$ is a proportionality factor that depends only on the temperature of the considered liquid (known as 'dynamic viscosity', formally linked to the kinematic viscosity by the relationship $\mu=\rho v$ where $\rho$ is the fluid density). Liquids satisfying this model are generally known as "Newtonian fluids" and the stresses modeled via the second term at the right-hand side of eq. (2) are known as "viscous stresses".

Though this simplification may seem natural at first sight, we must warn the reader that molten plastic materials cannot be described in the framework of such a relatively simple model. The peculiar properties displayed by plastics in the molten state are the natural consequence of the unique molecular structure of these materials. Plastics are polymers of very high molecular mass. Though they are often enhanced through the addition of specific additives, such as fillers and reinforcements, anti-degradants and stabilizers, flame retardants and plasticizers, the main attributes of a plastic material are essentially determined by the polymer it is mainly made of. Polymers, in turn, are macromolecules displaying a configuration where a large number of similar structural units are bonded together. A related category of liquids is that of "polymeric fluids", i.e. a Newtonian fluid (solvent) that acquires elastic properties due to the presence of dispersed polymer molecules. 
A statistical approach like that developed before for the simplified case of Newtonian liquids, cannot therefore be separated from proper consideration of the structure of such molecules and their ability to display in some circumstances 'elastic' properties.

The simplest way to represent mathematically such molecules is to consider the so-called Dumbbell configuration, i.e., a system formed by a spring connected to two beads, assumed to mimic a molecule that can generate and retain an elastic force when it is placed inside a Newtonian solvent (Bird et al., 1987, Bonito et al., 2011). The approach elaborated before for the Newtonian case can then be duplicated by applying separately the Newton's law to the two beads and accounting for the spring force and the drag that each of the two beads can experience as a result of its interaction with the solvent. In particular, the Newton's law applied to a dumbbell molecule can be formally written for each of two beads $(i=1$ and $i=2)$ having mass $m_{p}$ as:

$$
m_{p} \frac{d^{2} \underline{X}_{i}}{d t^{2}}=\xi\left[\underline{V}\left(\underline{X}_{i}, t\right)-\frac{d \underline{X}_{i}}{d t}\right] \pm \underline{F}(\underline{Q}(t))+\underline{R}_{i}(t)
$$

with the sign + or - being taken for $\mathrm{i}=1$ or $\mathrm{i}=2$, respectively. The meaning of the different quantities appearing in this equation can be illustrated as follows: the vectors $\underline{X}_{1}(t)$ and $\underline{X}_{2}(t)$ represent the position of the two beads with respect to an arbitrary fixed reference frame, the related difference $Q(t)=\underline{X}_{1}(t)-\underline{X}_{2}(t)$ can therefore be used to characterize the orientation in space of the overall polymer molecule and its 'internal' configuration. Moreover, the first and the second contributions at the right hand side of eq. (4) account for the drag force experienced by each bead (where $\xi$ is the 'drag coefficient'), and the elastic force $F$ associated with the deformation of the spring, respectively. From a purely physical standpoint, the last contribution $\underline{R}_{\mathrm{i}}(t)$ is due to the thermal agitation process represented by the microscopic velocities $\underline{C}$ previously introduced for the Newtonian liquid case.

The additional stresses created in the fluid by these mechanisms can formally be expressed as

$\underline{\underline{\tau}}=-\left\langle\sum_{j=1}^{N_{p}}\left[\underline{F}_{j} \underline{Q}_{j} \delta\left(\underline{r}-\underline{X}_{j}^{c m}\right)\right]\right\rangle$

where $\underline{X}^{c m}=\left(\underline{X}_{1}+\underline{X}_{2}\right) / 2$ is the center of mass of the molecule and $\mathrm{N}_{\mathrm{p}}$ is the number of polymer molecules in the fluid (see, e.g., Kramers, 1944, Giesekus, 1966). By assuming the dependence of the elastic force on $Q$ to be linear, the resulting Hookean dumbbell model (formally represented by eq. (5) in which $\underline{F}$ is replaced by $\mathrm{H} Q$ where $\mathrm{H}$ is the spring stiffness or Hook modulus) gives rise to a class of macroscopic viscoelastic models, generally known under the heading of Oldroyd-B. For such a case $\underset{\underline{\tau}}{\underline{\tau}} \approx-\left\langle\sum_{j=1}^{N_{p}}\left[\underline{Q}_{j} \underline{Q}_{j} \delta\left(\underline{r}-\underline{X}_{j}^{c m}\right)\right]\right\rangle$. Integrating this quantity via a distribution function and using eq. (4) to express the increment $d Q$ appearing in such integrals, formally yields a macroscopic transport equation (see, e.g., the related derivation given by Revuz and Yor, 1994) governing the 
evolution in time of $\underset{\underline{\tau}}{\underline{\tau}}$. This equation can be cast in compact form as (Alves et al., 2003 and references therein)

$$
\lambda\left(\frac{\partial \underline{\underline{\tau}}}{\partial t}+\underline{V} \cdot \underline{\nabla} \underline{\underline{\tau}}\right)+\underline{\underline{\tau}}=\eta\left(\underline{\nabla} \underline{\underline{\nabla}}+\underline{\nabla} \underline{V}^{T}\right)+\lambda\left(\underline{\underline{\tau}} \cdot \underline{\nabla} \underline{V}+\underline{\nabla} \underline{V}^{T} \cdot \underline{\underline{\tau}}\right)
$$

where $\lambda=\xi / 4 \mathrm{H}$ represents the so-called relaxation time of the viscoelastic fluid and the viscosity of the polymer $\eta$ can be expressed as $\left(2 \mathrm{n} \lambda \mathrm{H} a^{2}\right)=\left(\mathrm{n} \xi a^{2} / 2\right)$, n being the concentration of polymer molecules and $a$ the radius of gyration of the considered molecule.

Leaving aside for a while the related mathematical implications, this equation can be used to reveal the intimate essence of viscoelastic liquids. While simple (Newtonian) viscous liquids show a direct proportionality between shear stress and the rate of deformation (as formalized by eq. (2)), viscoelastic liquids are characterized by a time-dependent response to deformation. If they are subjected to a gradient of velocity, in addition to the classical frictional shear stress, a shear stress of non viscous nature builds up as a function of time (as formalized by eq. (6)). If the shearing is stopped, these additional stresses do not vanish immediately (as it would be the case for the viscous ones). Rather they relax with time and this process typically scales with $\lambda$, i.e. the relaxation time of the considered liquid.

Though eq. (6) was originally introduced to characterize highly elastic solutions consisting of a polymeric solute in a Newtonian solvent (the so-called 'Boger fluids'), it represents the parent of a vast category of more sophisticated models that have been elaborated over the years to deal with a variety of polymeric liquids and polymer melts (Saengow et al., 2015). It is also at the basis of the majority of the existing studies on thermal convection driven by gravity or surface tension effects, reviewed in Sects. 6-8. For these reasons and in order to fix the ideas, in the following we will always refer to this model (Oldroyd B).

As we shall show afterwards, most of the existing investigations have been parameterized in terms of the so-called "Elasticity number" (also known as Deborah number), a non-dimensional parameter proportional to the product of the fluid thermal diffusivity and its relaxation time and inversely proportional to the square of a characteristic length $(L)$ of the considered geometry, i.e.

$\theta=\frac{\alpha \lambda}{L^{2}}$

Another important parameter is

$$
\beta=\frac{\mu}{\mu+\eta}
$$


i.e. the ratio of the dynamic viscosity of the solvent and the "total viscosity" expressed as a summation of $\mu$ and the viscosity of the polymer $(\eta)$. This ratio is also used to define the so-called generalized Prandtl number

$\operatorname{Pr}_{\mathrm{g}}=\operatorname{Pr} / \beta$

which obviously reduces to $\operatorname{Pr}$ for standard Newtonian fluids $\left(\beta=1 \rightarrow \operatorname{Pr}_{\mathrm{g}}=\operatorname{Pr}\right)$.

Having completed a description of the different microscopic and macroscopic behavior of Newtonian and viscoelastic fluids, respectively, we can now move one step forward and consider the response of these fluids for different values of $\theta$ and $\beta$ when they develop the different forms of natural (induced by gravity or gradients of surface tension) convection mentioned in the introduction. Before doing so, however, it is necessary to introduce (briefly) the mathematical relationships that are generally used to link the variations of density and surface tension in a liquid to corresponding changes in the temperature. These models are general and do not depend on the specific type of fluid considered. We will also use them as an opportunity to define the additional non-dimensional numbers that fluid physicists and engineers use to characterize these types of convection.

\section{Gravitational effects and the Boussinesq Model}

From a mathematical point of view, the buoyancy force responsible for the onset of thermogravitational flow can be expressed as the product of the density of the considered liquids and the gravity acceleration $g$, namely:

$\underline{F}_{b}=\rho \underline{g}$

For most of liquids, the density can directly be expressed in terms of the fluid temperature as follows:

$\rho \cong \rho_{0}\left[1-\mathfrak{R}_{T}\left(T-T_{R E F}\right)\right]$

where $\mathfrak{R}_{T}$ is simply known as the thermal expansion coefficient and $T_{R E F}$ is a reference value for temperature, i.e. $\rho_{0}=\rho\left(T_{R E F}\right)$. Though the density of liquids can generally be considered constant (liquids are generally regarded as "incompressible" fluids), the dependence of their density on temperature must necessarily be retained in the buoyancy force (i.e. eq. (10)) in order to properly account for the physical mechanisms leading to the onset of thermogravitational convection. This approximation is generally known as the Oberbeck-Boussinesq model (Oberbeck, 1879 and Boussinesq, 1903). It has proven to be extremely accurate for many flows (Lappa, 2009, 2012). 
From a mathematical point of view, the thermal expansion coefficient appearing in eq. (10) can formally be seen as the first derivative of density with respect to temperature. From a physical standpoint, like the fluid thermal diffusivity and kinematic viscosity, it should be regarded as a thermodynamic property of the considered liquid. Most importantly, it contributes to the definition of the nondimensional number generally used to characterize thermogravitational convection, namely the so-called Rayleigh number (which measures the relative importance of buoyancy with respect to thermally diffusive and viscous effects). It is typically introduced as

$$
R a=\beta \frac{g \Re_{T} \Delta T L^{3}}{v \alpha}
$$

\section{Surface tension driven effects and shear stress balance}

The conditions for which a system of two immiscible fluid phases with their 'interphase layer' can be treated as two distinct volume phases separated by an idealized boundary with no thickness, i.e. an "interface" were examined in a consistent and exhaustive way by Napolitano (1979). As shown in that study, for many cases of practical interest, the surface tension along such a boundary can be assumed to behave as a linear function of the temperature (through a mathematical relationship formally similar to that already considered in Sect. 3 for the case of the density), i.e.

$\rightarrow \sigma \cong \sigma_{0}\left[1-\sigma_{T}\left(T-T_{R E F}\right)\right]$

where $\sigma_{\mathrm{o}}$ is the surface tension for $T=T_{R E F}\left(T_{R E F}\right.$ is a reference value), $\sigma_{\mathrm{T}}=-\mathrm{d} \sigma / \mathrm{d} T>0$ (which implies that $\sigma$ is a decreasing function of $T$ ). Originally introduced for Newtonian fluids, this approximation has been shown to be valid also for polymer molecules (Dee and Sauer, 1998 and references therein).

If a Newtonian liquid with nonisothermal free surface is considered, then the ensuing surfacetension forces $\underline{F}_{\sigma}^{T}=\underline{\nabla}_{S} \sigma$ (where $\underline{\nabla}_{S}$ represents the derivative tangential to the interface) are typically balanced by viscous stresses in the liquid (the equivalent viscous stresses in the gas surrounding the free liquid surface are generally ignored as they are almost negligible); from a mathematical point of view this balance reads:

$$
2 \mu(\underline{\nabla} \underline{V})_{o}^{s} \cdot \underline{\hat{n}}=-\sigma_{T}(\underline{\underline{I}}-\underline{\hat{n}} \hat{\hat{n}}) \cdot \underline{\nabla} T
$$

where $\underline{\hat{n}}$ is the unit vector perpendicular to the liquid/gas interface (directed from liquid to gas) and $I$ is the unity matrix. If the considered liquid is viscoelastic, the above relationship must obviously be enriched with the elastic stresses as follows: 
$\left[2 \mu(\underline{\nabla} \underline{V})_{o}^{s}+\underline{\tilde{\tau}}\right] \cdot \underline{\hat{n}}=-\sigma_{T}(\underline{\underline{I}}-\underline{\hat{n}} \hat{\hat{n}}) \cdot \underline{\nabla} T$

The following nondimensional number is of particular importance in such a context (see, e.g., Lappa, 2009):

$M a=\beta \frac{\sigma_{T} \Delta T L}{\mu \alpha}$

Generally known as the Marangoni number, it measures the relative importance of surface tension effects with respect to thermally diffusive and viscous effects.

\section{Governing equations, dissipative systems and bifurcations}

As a result of the physical arguments, microscopic interpretations and models elaborated in the preceding sections, the governing equations for mass, momentum, viscoelastic stress (using for simplicity the classical Oldroyd-B paradigm introduced in Sect. 2) and energy of a polymeric fluid can be cast in compact non-dimensional form as:

$\underline{\nabla} \cdot \underline{V}=0$

$\frac{\partial \underline{V}}{\partial t}+\underline{\nabla} \cdot[\underline{V} \underline{V}]=-\underline{\nabla} p+\operatorname{Pr} \nabla^{2} \underline{V}+\operatorname{Pr} \underline{\nabla} \cdot \underline{\tilde{\tau}}+\operatorname{Pr}_{g} R a T \underline{i}_{g}$

$\theta\left(\frac{\partial \underline{\underline{\underline{\tau}}}}{\partial t}+\underline{V} \cdot \underline{\nabla} \underline{\underline{\tau}}\right)+\underline{\underline{\tau}}=\zeta\left(\underline{\nabla} \underline{V}+\underline{\nabla} \underline{V}^{T}\right)+\theta\left(\underline{\underline{\tilde{\tau}}} \cdot \underline{\nabla} \underline{V}+\underline{\nabla} \underline{V}^{T} \cdot \underline{\underline{\tau}}\right)$

$\frac{\partial T}{\partial t}+\underline{\nabla} \cdot[V T]=\nabla^{2} T$

This non-dimensional formulation follows from the adoption of specific reference quantities for the various physical quantities appearing in the equations, namely $\alpha / L$ as reference velocity, $L^{2} / \alpha$ as reference time, $\rho \alpha^{2} / L$ as reference pressure, $\Delta T$ as reference temperature and $\rho v \alpha / L^{2}$ as reference viscoelastic stress.

If a free surface is involved in the process of interest, the additional equation for the stresses at the interface must be considered, i.e. eq. (13b). In particular, in non-dimensional form the related balance of viscous, elastic and surface-tension-driven stresses simply reads:

$\left[2(\underline{\nabla V})_{o}^{s}+\underline{\tilde{\tau}}\right] \cdot \underline{\hat{n}}=-M a(\underline{\underline{I}}-\underline{\hat{n}} \hat{\hat{n}}) \cdot \underline{\nabla} T$ 
Although this mélange of equations and related boundary conditions is necessary to derive quantitative results (and actually all the numerical results presented in the following sections have been obtained using these equations in their original non-linear version, as reported above, or in various simplified forms), in the following we will avoid detailed mathematics and attempt to look directly at the physical processes involved in the considered phenomena.

Along these lines, it is worth starting from some relatively simple notions, which are extremely important for a proper presentation and characterization of all these dynamics. These are the concepts of instability, bifurcations and transition to chaos. As we will see, these definitions can in turn be linked to another fundamental idea or realization in science, that is, when energy is injected into a system, this will tend to absorb it by activating different degree of freedom in space and in time, i.e., by developing a specific patterning behavior.

In such a context, it is also worth highlighting that fluid-dynamic processes are a special case of non-linear systems known as dissipative phenomena because the energy that they receive is somehow dissipated (in general due to frictional effects) and therefore a forcing mechanism is needed to restore it (to keep the considered process alive). In practice, when an imbalance is created in these counteracting effects, a bifurcation or instability can be produced, i.e. the system evolves from an initial configuration into a new equilibrium state that is generally less ordered than the previous one (due to breaking of symmetry in space and/or time).

In the following, these more or less abstract concepts are put in practice by reporting a series of focused examples intentionally selected after sifting through studies with different foci available in the literature. Towards the end to glean hints and draw inferences about the most significant aspects, we could successfully identify a number of key features spread in various numerical papers (which would take a relatively long time to be collected for those who wish to get in touch with the field). For the convenience of the reader these investigations are reviewed in the remainder of this chapter following a logical (historical perspective), and putting the necessary emphasis on the most important findings.

In particular, many of these papers have been based on linear stability analysis (LSA), i.e. a specific numerical approach where the original set of governing equations (eqs. (16)-(19)) is replaced by a linearized version of it, and the overall problem is reduced to the determination of the values of the characteristic numbers for which a bifurcation can be produced (the interested reader being referred, e.g., to Lappa, 2009 for additional details).

Perhaps, the best way to start to deal with an overview of all these efforts is to consider that, although the first studies on thermal convection date back to more than 100 years ago (Rayleigh, 1880), the vast majority of these investigations have been dedicated to Newtonian fluids. As explained before, only recently, have the mathematical models (and related computational techniques) about viscoelastic fluids reached a 'sufficient' level of maturity (in the sense that numerical techniques have become able to convey valuable and relevant information concerning the most important scientific questions relating to these problems). 
In line with historical developments, in the next sections the main properties of thermogravitational and surface-tension driven convection in viscoelastic liquids are discussed through a focused comparison with the equivalent behaviors in Newtonian fluids. Using what is known for Newtonian fluids as a basis, we move through a series of cases and situations specifically conceived to support reader's understanding of what happens when fluid elasticity enters the dynamics.

\section{Rayleigh-Bénard Convection: Historical perspective and review of recent results}

\subsection{Newtonian fluids}

As anticipated in Sect. 1, fluids uniformly heated from below give rise to "Rayleigh-Bénard" (RB) convection (Lord Rayleigh, 1916), one of the most intensively studied problems in fluid-dynamics due to the countless situations in nature and applications in technology where this type of flow can be encountered.

It is known from the experiments that by increasing progressively the temperature at the bottom of a fluid layer, the inherently unstable arrangement of heavy fluid over light fluid (produced as a result of the differential heating in the vertical direction), at a certain stage breaks down enabling fluid motion. What appears is a regular distribution of alternatively clockwise and anti-clockwise oriented parallel rolls, like the distribution shown in Fig. 1. Typically, for relatively small values of $\Delta T$, these rolls are steady.

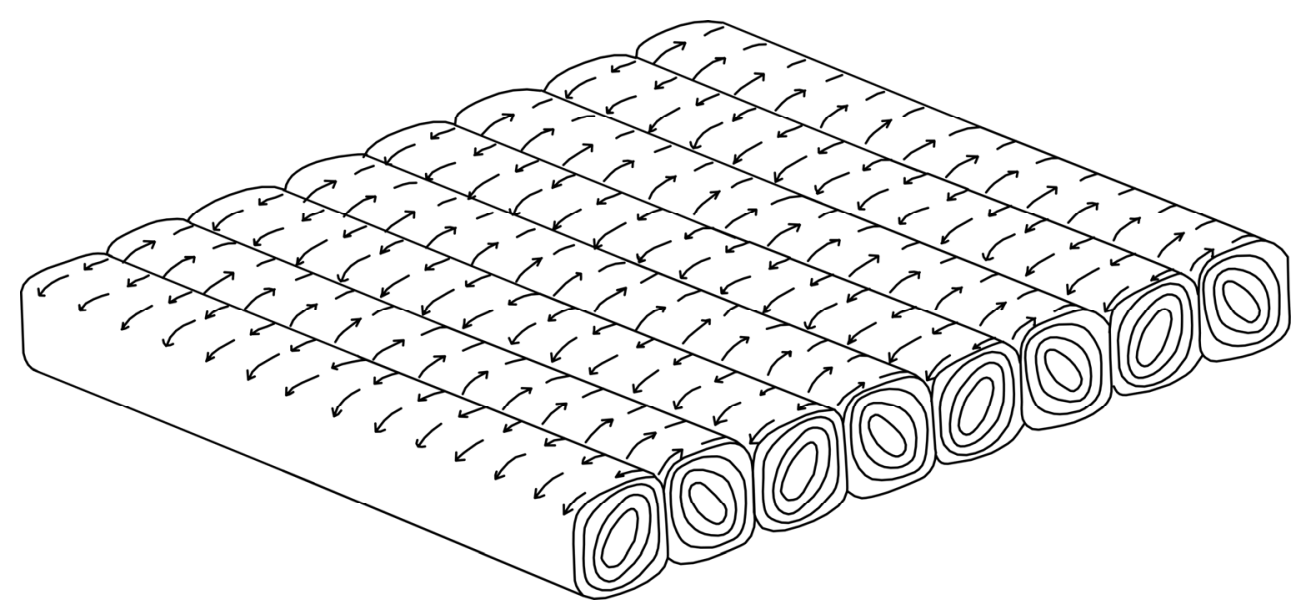

Figure 1: Sketch of parallel rolls typical of RB convection.

Such a stationary pattern is established by virtue of a delicate balance attained between the force driving fluid motion (thermal buoyancy), the dissipation of energy due to viscous effects and the diffusion at molecular level of heat. This is the reason why these patterns are also referred to as "dissipative structures" (see Sect. 5). Put simply, while buoyancy forces being active in the warmer fluid support the onset of fluid motion (RB instability), the viscous forces and thermal diffusion of the fluid tend to counteract it. These simple physical arguments may be regarded as a theoretical 
basis for the known dependence of these phenomena on the Rayleigh number (eq. (11)). For an infinite layer of a Newtonian fluid delimited from above and from below by solid walls, the minimum value of the Rayleigh number required to induce convection (critical Rayleigh number) is $\cong 1700$. This universal value does not depend on the Prandtl number. If the effective $R a$ is larger than this threshold, convection is produced, whereas if it is smaller than this limit the fluid remains in a quiescent state in which heat is transported only due to diffusion (the so-called thermally diffusive state).

The flow with regular parallel rolls (Fig. 1) emerging from the thermally diffusive quiescent state is generally referred to as the primary mode of convection.

This initial very simple convective structure can be turned into a more complex flow configuration by further increasing the Rayleigh number. When a second threshold is exceeded the flow enters a new state known as "secondary mode of convection" (named in this way to distinguish it from the first regime produced as a result of the transition from a quiescent to a convective state).

In reality, a set of distinct secondary instabilities exists, which can be categorized according to the different spatial symmetries that they break and the nature (stationary or oscillatory) of the new (emerging) convection. This multiplicity has led over the years to the introduction of a variety of colorful names, specifically conceived in the attempt to characterize in a synthetic way the main observable properties of the secondary flow. Examples are traveling waves, ziz-zag, cross-roll, bimodal, skewed, blob instability, traveling waves, etc. A detailed description of all these modes can be found in the book by Lappa (2009).

Most of these secondary flows are time-dependent, i.e. they imply a bifurcation from the initial steady state with perfect parallel rolls to a different less symmetric state in which fluid motion oscillates in time. The value of the Rayleigh number for which the secondary instability occurs essentially depends on the Prandtl number of the considered fluid (Lappa, 2009).

\section{$\underline{6.2 \text { Viscoelastic liquids }}$}

While RB convection in Newtonian fluids has been extensively studied both experimentally and numerically, knowledge about the typical primary and secondary modes of this type of convection in viscoelastic liquids is relatively limited.

Fundamental research on this specific topic has been conducted on an academic and industrial level over the last two decades, somewhat sporadically. As such, rather than a systematic and comprehensive review, what follows should be regarded as a focused overview of key aspects.

In this regard, it is worth starting from what can be seen as the hallmark of RB convection in viscoelastic fluids, namely its ability to emerge at a Rayleigh number that is smaller than that predicted for the corresponding stationary convection in Newtonian fluids.

This line of studies was pioneered by Green (1968), Vest and Arpaci (1969), Sokolov and Tanner (1972) where for the first time it was shown that the lowering of the critical Rayleigh number in these cases must directly be ascribed to the competition between the processes of viscous relaxation 
and thermal diffusion. These initial studies were obtained in the framework of the aforementioned linear stability analysis approach (LSA). Other important results were yielded later by Kolkka and Ierley (1987), Martínez-Mardones and Pérez-Garcí1a (1990) and Larson (1992), where it was shown that viscoelastic forces can enable completely new mechanisms for instability that are physically impossible in Newtonian liquids. In the following, in particular, we refer directly to the categorization of these phenomena elaborated by Li and Khayat (2005).

According to such meaningful classification, the dynamics of RB in viscoelastic fluids can be split in two main branches or areas, namely the weakly elastic regime (WER) and the strongly elastic regime (SER).

For a fixed viscosity of the solvent and of the polymer (i.e. for a fixed value of the parameter $\beta$ defined by eq. (8)), these two branches of solutions can be distinguished on the basis of the elasticity number defined via eq. (7). In particular, the former regime is attained in a range of relatively small values of $\theta$, namely for $\theta$ increasing from 0 (Newtonian liquid case) to a limiting value $\theta_{\mathrm{h}}$. In this interval, emerging convection is steady just like that produced in the purely Newtonian case. If the $\theta_{\mathrm{h}}$ threshold is exceeded (SER), however, there is a significant change in the nature of the primary flow, which becomes essentially oscillatory (this condition is generally referred to as "overstability", see, e.g., Li and Khayat, 2005). One of the most remarkable achievements obtained in recent years is indeed the realization that for most of cases of practical (technological) interest, RB convection originating from the initial quiescent state of viscoelastic fluids is essentially oscillatory.

The different curves reported in Fig. 2 substantiate qualitatively and quantitatively the dependence of the neutral Rayleigh number (representing the transition from the quiescent state to convection) on the wavenumber (q). They are typical examples of LSA-based results. The wavenumber (see, e.g., Lappa, 2009) is defined for these problems as $q=2 \pi / \ell$ where $\ell$ is the wavelength of the pattern, i.e. two times the expected nondimensional diameter of the emerging convection cells (ratio of the horizontal cell extension to the depth of the liquid layer). The minimum of these curves represents the aforementioned 'critical' threshold above which convection is effectively enabled. In turn, the related value of q can be used to predict the aspect ratio of the emerging rolls.

For Newtonian fluids (solid line in Fig. 2), as explained before, the entire neutral curve is known to be independent of Pr. Its minimum corresponds to $R a_{c r}=1707$ and $\mathrm{q}_{\mathrm{cr}} \cong 3.12(\pi / 3.12 \cong 1.007$, which makes the predicted horizontal size of the rolls very close to the height of the layer).

However, for a fixed $\beta$, a value of $\theta$ exists, for which the minimum of the neutral curve for the viscoelastic fluid becomes smaller than the value needed for the onset of (steady) convection in the Newtonian case. This value corresponds to the aforementioned $\theta_{\mathrm{h}}$ separating the SER and WER regimes. For $\theta>\theta_{\mathrm{h}}$, oscillatory convection will be produced first and, as evident in the sketch, it will typically feature a slightly larger value of the wavenumber q (leading to rolls with smaller size). For $\theta<\theta_{\mathrm{h}}$, oscillatory flow is still possible, however, it typically emerges as a result of a secondary instability of the pre-existing stationary convective states (produced for $R a>1707$ ) and requires a 
value of the Rayleigh number larger than that needed to get steady convection in the Newtonian case.
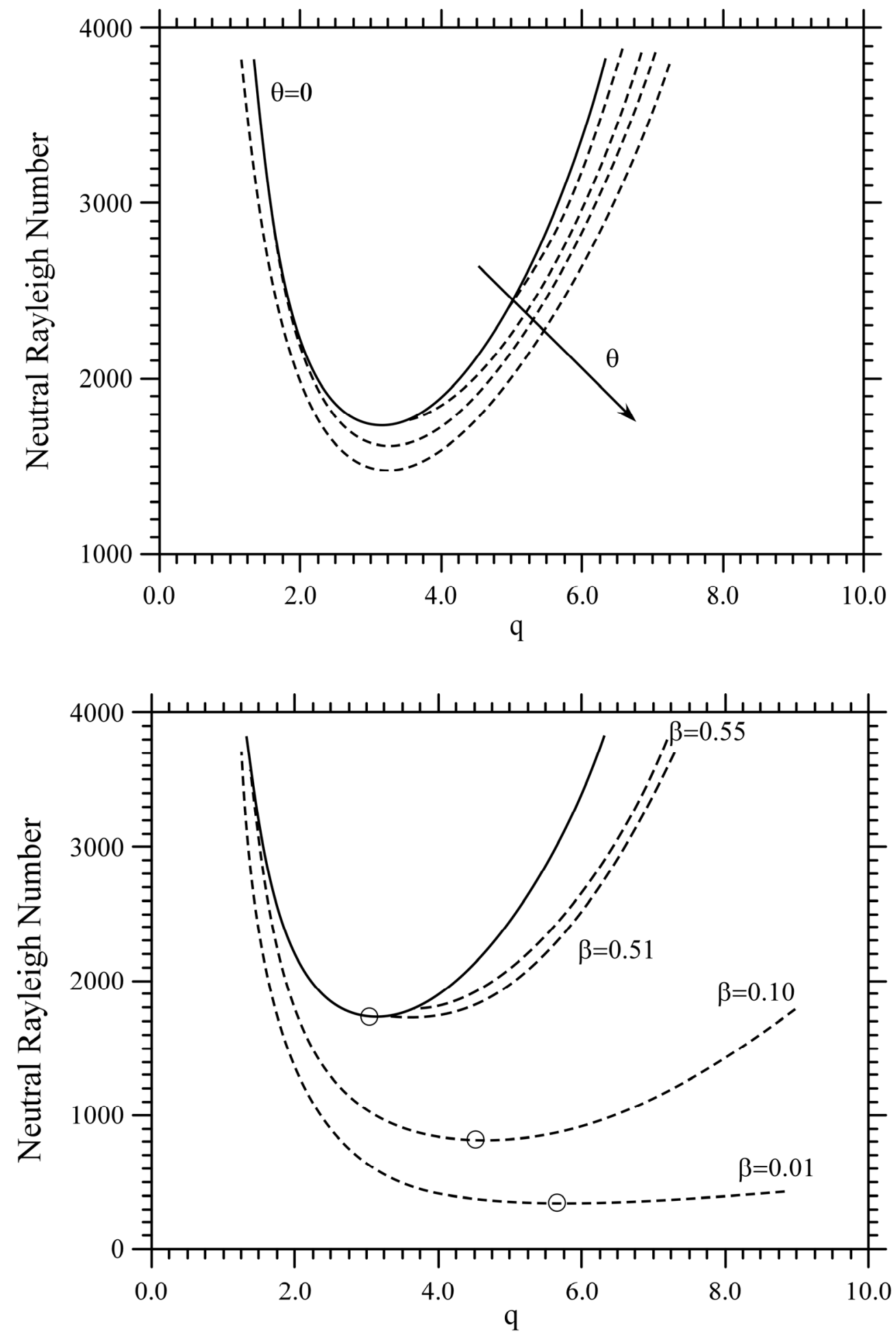

a)

b)

Figure 2: Neutral Rayleigh number as a function of the wavenumber q (layer limited from above and from below by isothermal differentially heated walls, $R a$ based on the depth $\mathrm{d}$ of the layer): a) Sketch showing the influence of $\theta$ for a fixed value of $\beta$; b) LSA results for $\operatorname{Pr}_{\mathrm{g}}=10, \theta=0.1$ and several values of the parameter $\beta$ (the solid curve refers to stationary convection, the dashed curves to oscillatory flow, $\theta=\alpha \lambda / \mathrm{d}^{2}, \beta=\mu /(\mu+\eta)$; after Martínez-Mardones and Pérez-Garcí1a, 1990, (C) IOP Publishing, reproduced with permission, all rights reserved). 
Unlike the Newtonian case, for $\theta>\theta_{\mathrm{h}}$, the minimum value of the Rayleigh number needed for the onset of convection is not independent of Pr, i.e. it changes according to the considered viscoelastic fluid and, in general, (see Fig. 2a) it decreases with $\theta$. For a fixed $\operatorname{Pr}_{\mathrm{g}}$ and $\theta$, it also depends on the parameter $\beta$. This behavior can directly be gathered from Fig. $2 \mathrm{~b}$ where it can be seen that a decrease in $\beta$ plays essentially a destabilizing role (smaller values of $\beta$ correspond to a smaller $\theta_{\mathrm{h}}$ ). On the basis of the studies cited above, it is also known that $\theta_{\mathrm{h}}$ behaves as a decreasing function of $\operatorname{Pr}_{g}$.

Since LSA can provide meaningful data only about the threshold value of the parameter (Rayleigh number) and related wavenumber, whereas it cannot make predictions on the effective magnitude (amplitude) of the emerging convection (and its patterns), other authors have expanded this line of research through the application of other approaches such as the use of amplitude equations, the power-series method and other similar techniques. Relevant examples in this regard are the analyses by Eltayeb (1977), Rosenblat (1986), Martínez-Mardones and Pérez-Garcí1a (1992), Park and Lee (1996), Martínez-Mardones et al. (1996), Parmentier et al. (2000) and Li and Khayat (2005).

While some of these researchers have concentrated on the spatial pattern effectively formed by convection, other authors have tried to determine the prevailing behavior of the emerging oscillatory flow.

Most of the studies concerned with the first aspect, in particular, have considered the WER. A seminal work focused on this aspect was published by Parmentier et al. (2000). These researchers investigated the ability of RB to produce stationary convection with different types of symmetry (namely solutions with classical two-dimensional rolls like those typical of Newtonian fluids or alternate states with three-dimensional square cells or hexagonal cells) in layers of viscoelastic liquids heated at the bottom wall and limited from above by an adiabatic liquid-gas interface assumed to be stress-free (i.e. with no surface tension effects). Li and Khayat (2005) reconsidered the same problem expanding the set of possible boundary conditions. These authors found that stationary hexagonal cells, known to be unstable for the purely Newtonian case, can effectively be produced in a certain range of elasticity number. They also conducted a parametric analysis of the influence of the Prandtl number showing that the likelihood of these two- or three-dimensional patterns in typical polymeric solutions essentially depends on the viscosity ratio $(\beta)$.

For the other regime (SER) and aspects relating to oscillatory states, it is worth citing the study by Martínez-Mardones et al. (1996) where attention was specifically paid to determine the stability range of standing waves potentially emerging in the SER in proximity to the instability threshold. These specific waveforms can be distinguished from the classical travelling waves due to their peculiar spatio-temporal behavior, characterized by the presence of flow or temperature features that 'pulsate' at fixed positions in space (e.g., to fix the ideas the reader may imagine cold and hot temperature spots produced in specific points, which grow and shrink periodically in such a way that hot spots are replaced in time by cold ones and vice versa).

As shown by earlier studies about the secondary modes of convection of classical RB in Newtonian fluids, the signature of travelling waves is quite different. These specific convective modes typically 
manifest themselves as disturbances that move in the fluid (to fix ideas we may again consider hot and cold spots, which in this case, however, would travel continuously along a given direction in the liquid).

As evident after comparative readings of the works by Martínez-Mardones et al. (1996) and other articles, standing waves seem to be favored in viscoelastic RB convection with respect to traveling disturbances. Interestingly, some additional evidence along these lines has been provided by other numerical studies where the effect of lateral boundaries has been taken into account (the analyses cited above were essentially developed under the assumption of laterally unbounded, i.e. infinite layers). Like the case of the infinite layer, many authors have initially addressed finite-size geometries on the basis of LSA paying particular attention to the SER (first for a box of fixed aspect ratio, see Park and Ryu, 2001a, and then in domains with arbitrary finite size, Park and Park, 2004). Continuing with historical developments, it is also worth mentioning the later efforts where weakly non-linear effects were taken into account (Park and Ryu, 2001b; 2002; Lyubimov et al., 2011, 2012) and the more recent ones where the problem was finally addressed using finite-difference techniques applied to the governing equations in their fully time-dependent and non-linear form (Krapivina and Lyubimova, 2000; Park et al., 2009; Park and Lim, 2010; Kovalevskaya and Lyubimova 2011 and Park, 2018). Though all of these studies focusing on systems with finite lateral extension were carried out under the assumption of two-dimensional flow, interestingly, they could confirm that convection tends to emerge as a standing wave, namely, a periodic change in the sense of circulation of the flow. Very recently, by means of 3D simulations (Lappa and Boaro, 2020) it has been shown that if the constraint of two-dimensionality is removed, even more complex dynamics can be produced. Some numerical results along these lines are shown in Fig. 3.

Interestingly, it has been clarified that the set of alternatively clockwise and anti-clockwise oriented parallel rolls typical of steady RB convection can be taken over by a peculiar mechanism of oscillations where rolls continuously break and reform in time with a new orientation in space (which is essentially perpendicular to the original one). This behavior can clearly be seen in the sequence of snapshots reported in Fig. 3.

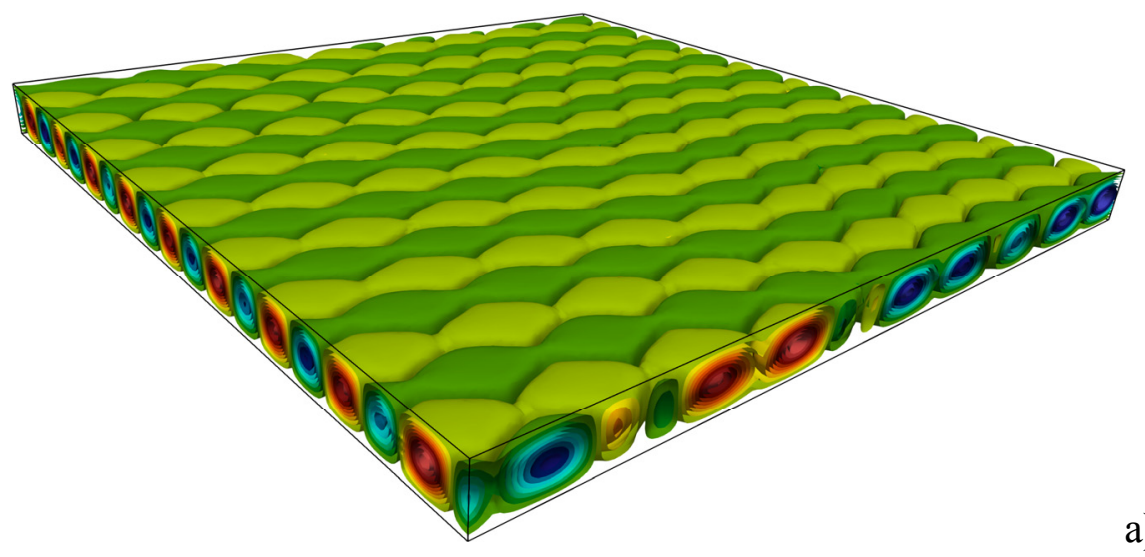

a) 


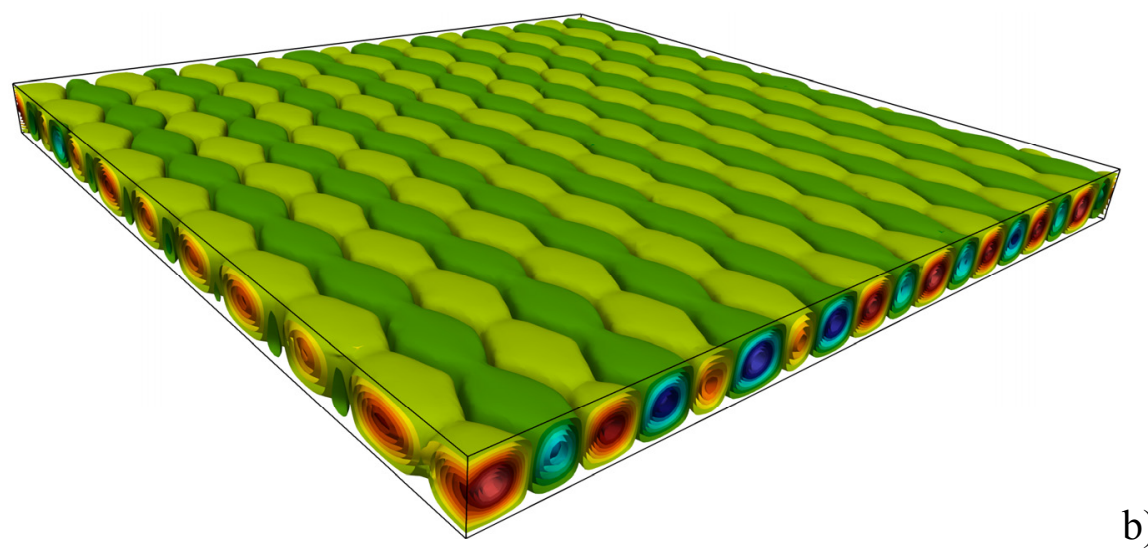

b)

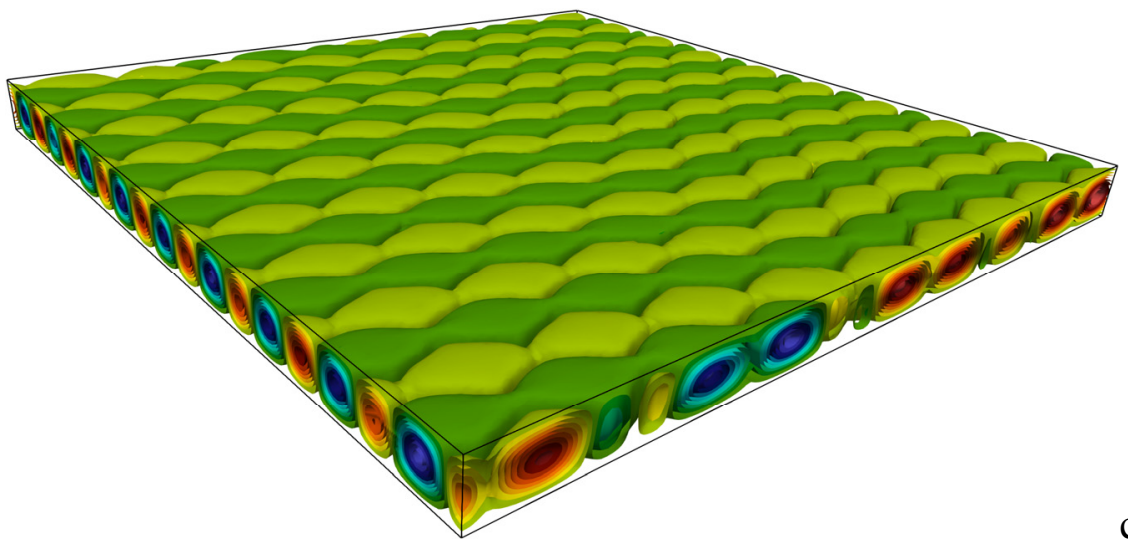

c)

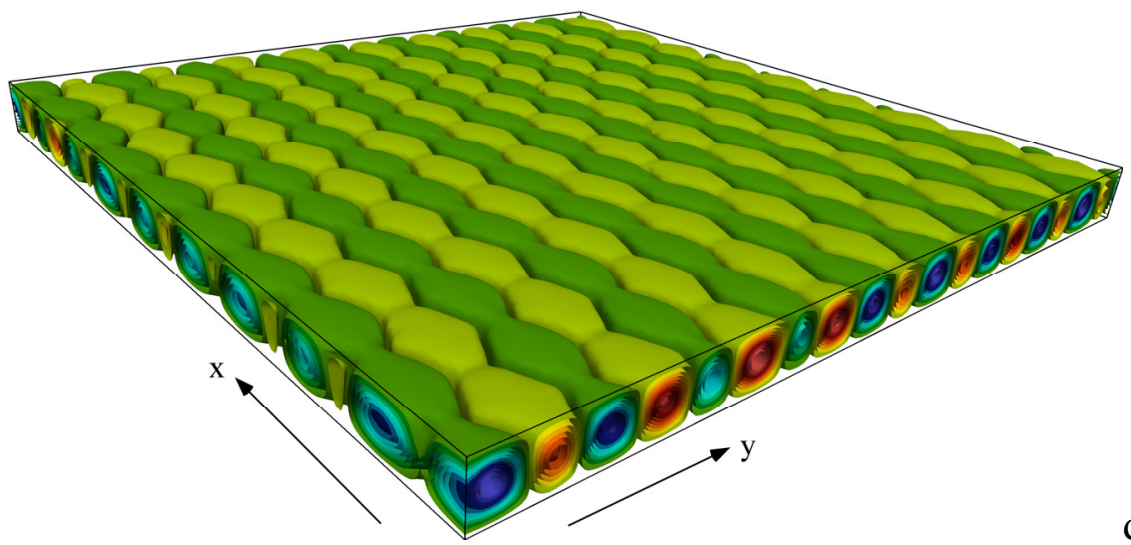

d)

\section{Axial Velocity}

$\begin{array}{lllllll}-4.9 & -4 & -2 & 0 & 2 & 4 & 4.9\end{array}$

Figure 3: Rayleigh-Bénard convection in a layer of viscoelastic fluid heated from below and cooled from above (snapshots of the isosurfaces of the vertical component of velocity evenly distributed over the oscillation period, $15 \times 15 \times 1$ domain with top and bottom solid walls and periodic lateral boundary conditions, $\beta=\mu /(\mu+\eta)=1 / 2, \operatorname{Pr}_{\mathrm{g}}=10, R a=2000, \theta=0.1$, elasticity number defined as $\theta$ $=\alpha \lambda / \mathrm{d}^{2}$ where $\mathrm{d}$ is the depth of the layer, non-dimensional angular frequency of oscillation $\omega \cong 9.2$; numerical simulation by Alessio Boaro).

While in the first snapshot (Fig. 3a) rolls are essentially aligned with the y axis, in Fig. 3b their axes are oriented along a perpendicular direction. In Fig. $3 \mathrm{c}$ the orientation changes again and the flow 
comes back to a configuration dominated by rolls horizontally extended along y. However, careful comparison of this snapshot with the equivalent one shown in Fig. 3a reveals that, even if the spatial orientation of rolls is the same, the positions which were previously occupied by rolls with clockwise sense of rotation are now featured by rolls with the opposite sense of rotation (and vice versa, thereby resembling the typical behavior of a standing wave). In Fig. 3d, rolls perpendicular to those shown in Fig. 3c re-emerge, and, accordingly, a situation with rolls parallel to $\mathrm{x}$ is recovered. Yet, it can be noticed that the flow configuration is not identical to that seen in Fig. 3b, rather it should be regarded as its mirror image (due to the opposite sense of rotation of rolls located at the same stations along y). On further increasing the time, a condition like that shown in Fig. 3a is attained and a new cycle of oscillation can start (with rolls that periodically break and form alternately elongated structures along $\mathrm{x}$ and $\mathrm{y}$ ). Three-dimensional studies based on the solution of the governing equations in their complete form have therefore revealed that the effective behavior can be more complex than that of a classical standing wave (due to the mechanism described above, involving a periodic reorientation in the prevailing direction of rolls).

\section{Marangoni-Bénard Convection: Typical planforms and "oscillons"}

\subsection{Newtonian fluids}

Like RB phenomena, also Marangoni-Bénard (MB) convection in Newtonian liquids has enjoyed a widespread interest over the last century. Actually the first observations of this type of flow date back to 1900 when Bénard $(1900,1901)$ reported on the emergence of suggestive and pleasing hexagonal patterns, which resemble the architecture created by bees for their honeycombs (Fig. 4). These planforms displaying a well-defined polygonal symmetry were initially misinterpreted by Rayleigh (1916) as the consequence of convective mechanisms similar to those driving RB convection. Almost 40 years later, however, Block (1956), gave a conclusive (experimental) demonstration of the crucial role that surface tension effects have in the formation of these patterns. In particular, this researcher managed to observe cells at $R a$ much smaller than the value needed to produce RB flow.

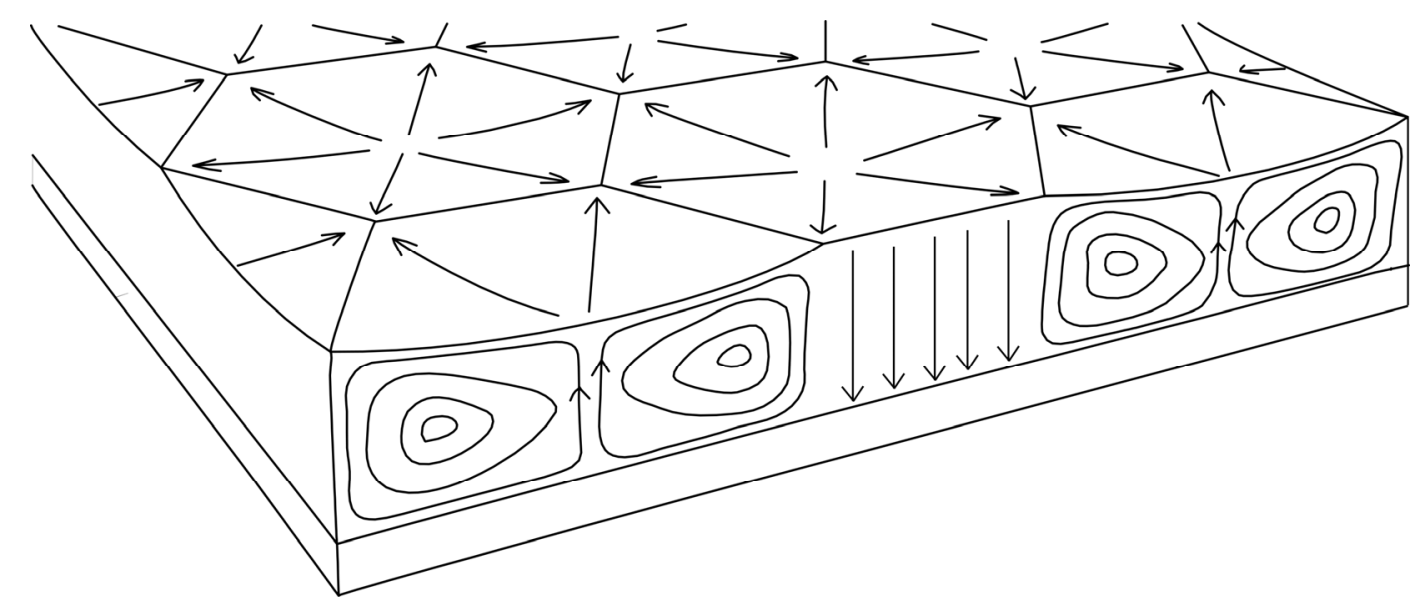

Figure 4: Sketch of the convective pattern with hexagonal symmetry typical of MB convection. 
Nowadays, a convective structure with the hexagonal symmetry (Fig. 4) is regarded as the typical signature of $\mathrm{MB}$ convection. Sometimes, this phenomenon is also referred to as the Pearson instability (after the LSA by Pearson, 1958). A large cross section of fundamental research on this topic has been reviewed by Colinet et al. (2001), and Lappa (2009). In this introductive section we limit ourselves to reporting some salient aspects which relate to Newtonian fluids only.

The pattern with steady aesthetic hexagonal cells is indeed a typical feature of Newtonian high-Pr liquids. Like RB convection, also this primary mode of convection is steady. If the applied temperature difference is increased, however, it is generally taken over by planforms with other symmetries. In particular, compelling evidence has been provided (see, e.g., Bestehorn, 1996 and references therein) that if the temperature difference (the Marangoni number) is increased, the dominant flow topology shifts from a hexagon-based symmetry to a new organization based on the coexistence of squares. This transition involves a topological process where the threefold vertices of the initial hexagonal cells become fourfold. Further increase in the Marangoni number can make this flow time-dependent.

Though the pattern with square symmetry is typically a secondary mode of convection for fluids with $\operatorname{Pr}>1$, it takes the role of primary mode if fluids with $\operatorname{Pr} \cong 1$ are considered. In this specific case, on increasing the temperature difference, the initial squares can even be turned into unsteady triangular cells (Lappa and Ferialdi, 2018).

\subsection{Viscoelastic fluids}

Studies about the dynamics of MB convection in viscoelastic liquids are relatively rare and sparse. As a fleeting glimpse into existing literature for the companion case of RB convection would reveal, however, these two types of flow share notable similarities.

Also in this case, for a fixed value of the non-dimensional parameter $\beta$ (eq. (8)), the steady primary modes of convection typical of Newtonian liquids are taken over by time-dependent solutions if a given threshold in terms of elasticity number $(\theta$, see eq. (7)) is exceeded (Getachew and Rosenblat, 1985; Lebon and Cloot, 1988; Lebon et al., 1994; Dauby et al., 1993; Siddheshwar et al., 2011). Moreover, the critical Marangoni number required for the onset of these oscillatory flows is smaller than the corresponding value for Newtonian fluids, which clearly indicates that the aforementioned concept of "overstability" also applies to these cases (see, e.g., Ramkissoon et al., 2006 and Hernández Hernández and Dávalos-Orozco, 2015).

This behavior can be seen in Fig. 5a. Following the same approach already undertaken for RB convection, we have included in this plot the neutral curve for the Newtonian fluids (solid line). In that case, assuming adiabatic conditions for the free surface, flow is produced as soon as the Marangoni number exceeds $\mathrm{Ma}_{\mathrm{cr}}=79.607$ and the related wavenumber is $\mathrm{q}=1.99$. When viscoelastic effects enter the dynamics, the general trends are relatively similar to those already illustrated for $\mathrm{RB}$ convection. A specific value of $\theta$ can be identified, which separates SER and WER states. 

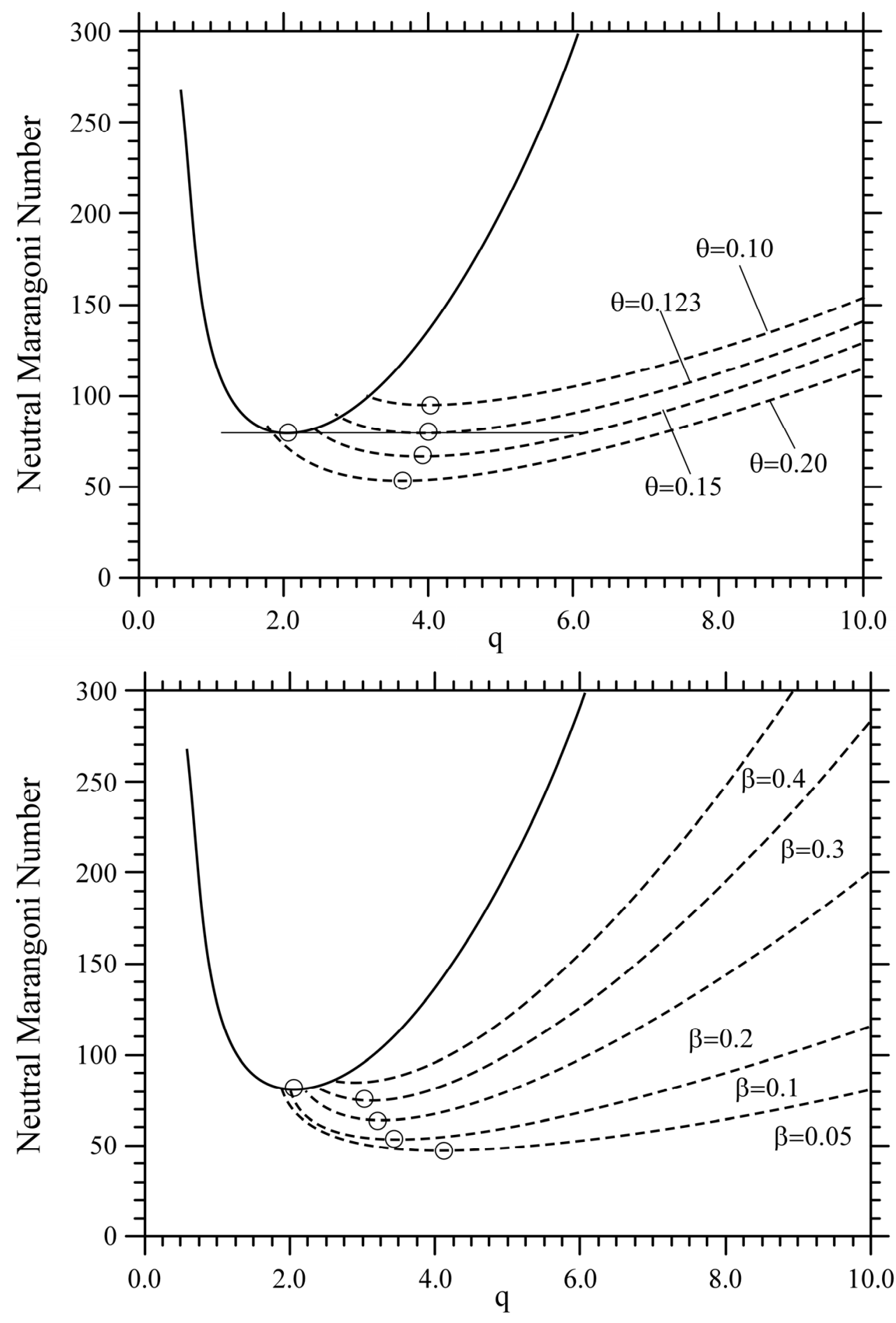

a)

b)

Figure 5: Neutral Marangoni number as a function of the wavenumber q (layer limited from above by an isothermal hot wall and from above by an adiabatic free surface, Ma based on the thickness of the layer) for $\operatorname{Pr}_{\mathrm{g}}=200$ and $R a=0$ (reprinted by permission from Springer Nature, Lebon et al., Rheol. Acta, 1994; the solid curve refers to stationary convection, the dashed curves to oscillatory flow): a) $\beta=0.1$ and several values of the parameter $\theta\left(\theta=\alpha \lambda / d^{2}\right)$, b) $\theta=0.2$ and several values of the parameter $\beta=\mu /(\mu+\eta)$.

This value depends on the considered fluid and $\beta$ (as an example, for $\operatorname{Pr}_{\mathrm{g}}=200$ and $\beta=0.1$, as shown in Fig. 5a, $\left.\theta_{\mathrm{h}}=0.123\right)$. For elasticity number taking values that are larger than this limit, the instability threshold is lowered and the convection emerging from the quiescent state is oscillatory 
(dashed lines). Remarkable changes can also be seen in the wavenumber, which increases significantly.

Overstability obviously is also sensitive to the parameter $\beta$ accounting for the relative importance of the different viscosities involved in the problem. As evident in Fig. 5b, for a fixed elasticity (constant value of $\theta$ ) overstability occurs only if the parameter $\beta$ is smaller than a given threshold $\left(\beta=0.3\right.$ for $\left.\operatorname{Pr}_{\mathrm{g}}=200\right)$. It can also be seen that the layer becomes progressively more stable as $\beta$ grows (the behavior of a Newtonian fluid would be ideally recovered in the limit as $\beta \rightarrow 1$ ).

As made evident by a comparative analysis of the trends displayed by RB and MB flows (compare Figs. 5 and 2), these two types of convection undergo quite similar dynamics when viscoelastic effects are considered. Overstability is indeed a common feature and related trends (in terms of critical parameters and their dependence on elasticity and viscosity ratio) display notable analogies. However, unlike RB convection, unfortunately only a few analyses have been devoted to the investigation of the overstable regime of MB flow taking into account non-linear effects. Relevant efforts along these lines have been provided by Wilson (1992) and Parmentier et al. (2000). Another relevant study worthy of mention is that by Lappa and Ferialdi (2018) where the problem has been addressed in the framework of direct numerical solution of the fully non-linear and time-dependent fluid flow balance equations. In particular, a kaleidoscope of possible states has been revealed by assessing the response of a fluid layer of a $\left(\operatorname{Pr}_{\mathrm{g}}=1\right)$ liquid to the progressive increase of the

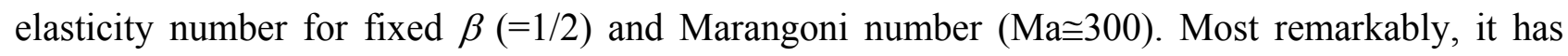
been demonstrated that these systems can undergo transition to chaos even for relatively small values of the driving force provided the elasticity of the fluid is sufficiently high (we will come back to this interesting concept in Sect. 9).

More specifically, Lappa and Ferialdi (2018) have illustrated that starting from the conditions corresponding to Newtonian fluid where the flow displays a very regular texture formed by the combination of almost triangular cells (this being the expected planform for the considered values of $\operatorname{Pr}$ and $\mathrm{Ma}$ ), on increasing the elasticity number, this pattern undergoes a progressive and fascinating 'corrugation' process that can turn the apparently crystalline (fixed) nature of the initial arrangement of cells into a much more flexible and dynamic behavior. The related evolution as a function of $\theta$ at fixed Ma can directly be appreciated by taking a look at Fig. 6.

It can be seen that as $\theta$ is increased starting from the situation $\theta=0$, corrugation initially manifests itself as a set of localized spatial defects in the flow topology, which alter the regular polygonal multi-cellular structure in some sub-regions of the physical domain (Figs. 6a and 6b). Such morphological alterations are also coupled with interesting time-dependent effects (see Lappa and Ferialdi, 2018 for additional details).

The higher the elasticity parameter, the larger the alterations affecting dynamics with respect to the Newtonian case (Fig. 6c). Moreover, some changes can also be spotted in the number of cells being present at the same time in the pattern. The spatial extension of the cells becomes increasingly larger while their number is reduced accordingly (Fig. 6d). Moreover, the initial configuration with triangular cells all rigidly embedded in a crystalline texture (side-by-side arrangement) is taken over 
by a new organization where different cells tend to be separated by intermediate regions of more or less motionless fluid (referred to as 'buffer' regions in Lappa and Ferialdi, 2018). As shown in Figs. $6 \mathrm{~d}$ and $6 \mathrm{e}$, on further increasing $\theta$ the size of these buffer regions grows and, paralleling this trend is a noticeable change in the shape of the convective cells, which tend to lose their polygonal appearance and be transformed into more or less rounded entities.

Most importantly, as a result of these modifications, the convective cells are provided with new degrees of spatial freedom which were not possible in their original (crystal-like) arrangement. Owing to the excitation of these new degrees of freedom (the reader being referred to Sect. 9 for further elaboration of this concept), MB convection finally enters a state resembling the chaotic motion of a group of 'islands' in the sea (Fig. 6e). Lappa and Ferialdi (2018) observed the cells in this state to move back and forth along uncorrelated horizontal directions. This specific behavior led them to refer to these excitations with the name of "oscillons", loosely used to indicate localized time-dependent convective structures in an otherwise uniform background (already known in the literature for their ability to produce turbulence in other types of convection through mutual interaction, Lappa, 2019a).
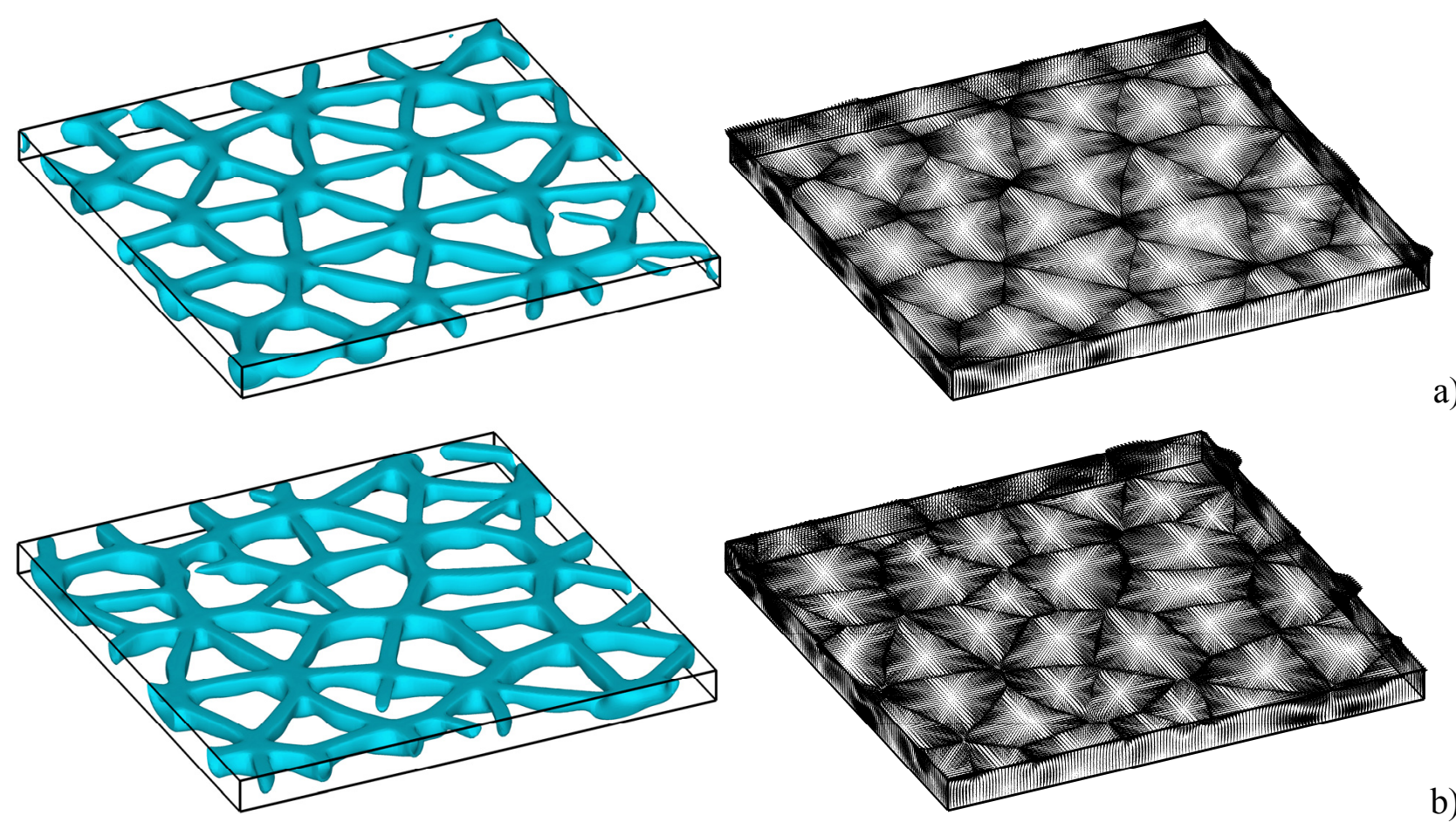

b)
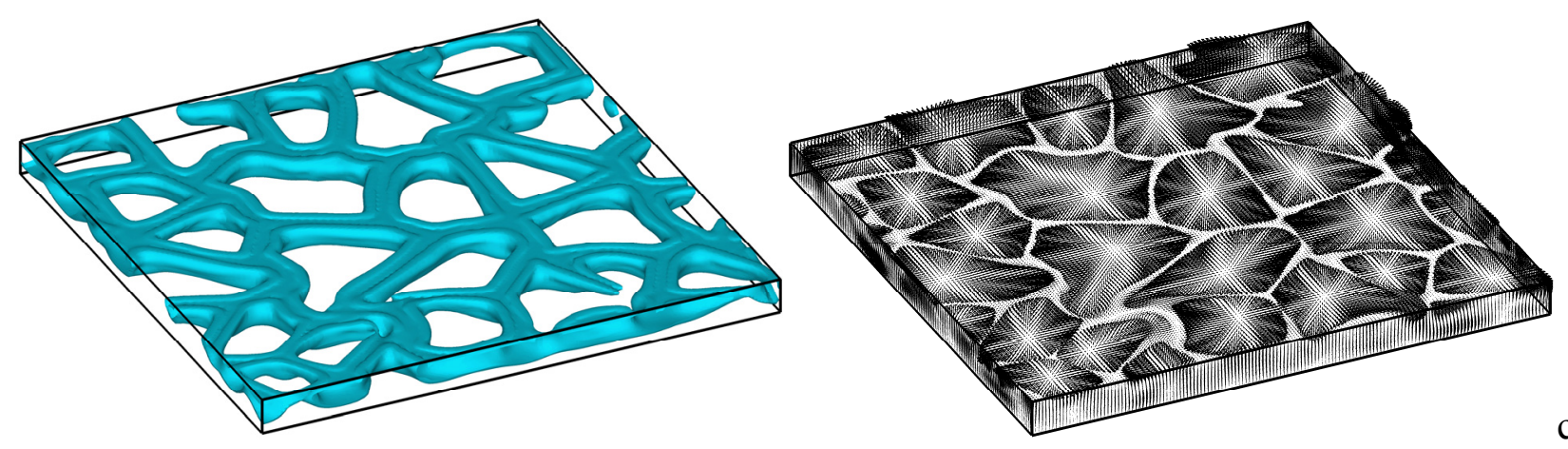

c) 

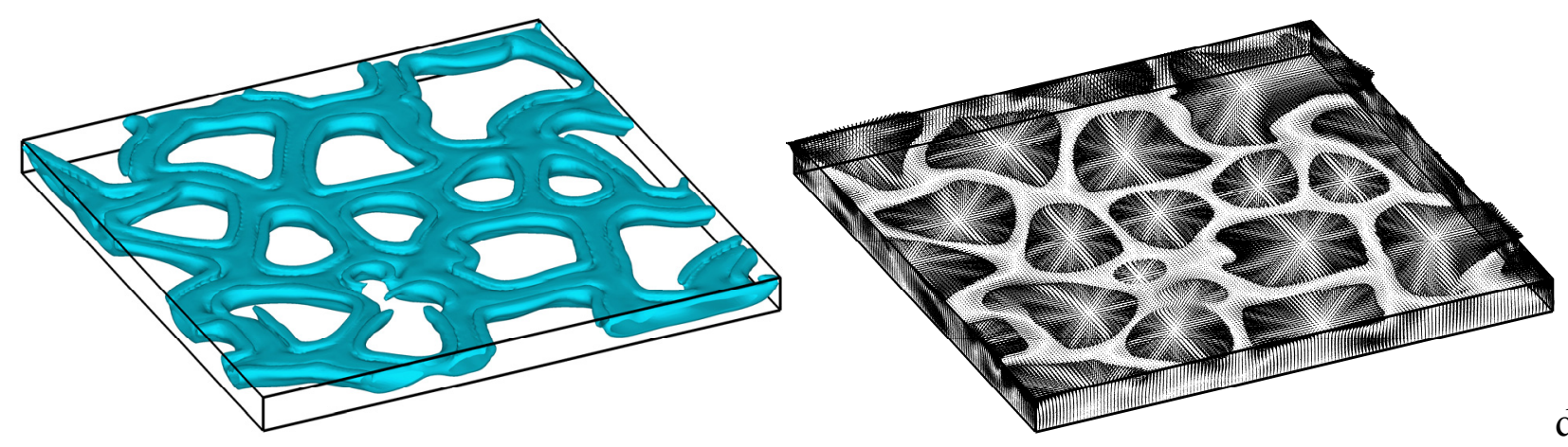

d)
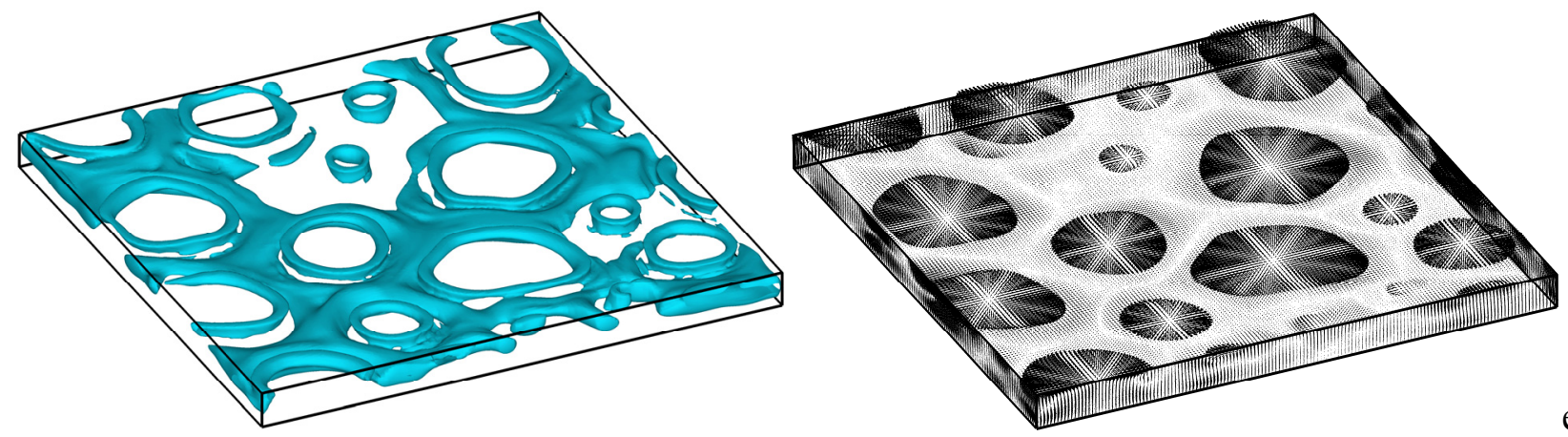

e)

Figure 6: Marangoni-Bénard convection in a layer of viscoelastic fluid heated from below (snapshot of the isosurfaces of the component of velocity perpendicular to the free surface and related vector field) for different values of the elasticity parameter showing the island-formation effect $\left(15 \times 15 \times 1\right.$ domain with periodic boundary conditions, $\beta=\mu /(\mu+\eta)=1 / 5, \operatorname{Pr}_{\mathrm{g}}=1, \mathrm{Ma}=300$, $\mathrm{Bi}=0.1$, elasticity number defined as $\theta=\alpha \lambda / \mathrm{d}^{2}$ where $\mathrm{d}$ is the depth of the layer): a) $\theta=0$ (Newtonian fluid), b) $\theta=7 \times 10^{-3}$, c) $\theta=3 \times 10^{-2}$, d) $\theta=5 \times 10^{-2}$, e) $\theta=9 \times 10^{-2}$.

\section{Marangoni (thermocapillary flow): From Hydrothermal waves to stationary rolls}

As explained to a certain extent in the introduction, a rotation of the direction of the temperature difference by $90^{\circ}$ with respect to the canonical MB system, causes a change in the flow nomenclature. The resulting fluid motion is generally simply known as "Marangoni" or "thermocapillary" convection. This observation, however, should not be misread as implying that the only outcome of the different direction of the $\Delta T$ is just a formal alteration of the terminology. Modifications are indeed substantial and concern both the nature of the flow and the related hierarchy of instabilities.

The first and most evident change is the ability of this type of convection to emerge regardless of the value taken by the Marangoni number. Put differently, this indicates that a quiescent (motionless) and thermally diffusive state is no longer a possible solution for this case. As soon as a temperature difference along the free interface is applied, fluid starts to move with no need to exceed a given threshold or limit.

In practice, if an extended layer of fluid is considered the (primary) flow generally consists of a current of fluid directed from the hot to the cold side along the interface and in the opposite 
direction along the bottom wall. Taken together these two currents give rise to what is generally known as the "return flow" solution (see, e.g., Lappa, 2009 for some related analytic treatment).

If the Marangoni (temperature difference) is relatively small and a Newtonian fluids is considered, this convective state is steady and regular (in finite-size containers it generally takes the shape of a single circulation elongated in the direction of the applied temperature gradient). If the Marangoni number is increased, however, this elongated roll undergoes an instability and is taken over by a new state that is oscillatory in time (secondary flow). A first milestone study along these lines, on which several numerical approaches would successively rely, was the LSA performed by Smith and Davis (1983).

These authors found that the return-flow solution described above can produce travelling waves, i.e. convective disturbances that travel continuously in the fluid.

In particular, these waves always consist of two components, one parallel to the temperature difference and another being directed in the perpendicular (spanwise) direction. The existence of these two components makes the direction of propagation of the resulting disturbance essentially inclined with respect to the direction of the temperature difference. The related angle is a function of the Prandtl number, i.e. the properties of the secondary flow depend on the considered liquid (Smith and Davis, 1983). In particular, for relatively high values of Pr, the wave inclination angle is relatively small, and the final outcome of the instability is a series of co-rotating rolls with axis almost perpendicular to the temperature difference, which travel in the same direction of the temperature gradient (from cold to hot regions), i.e. in the upstream direction.

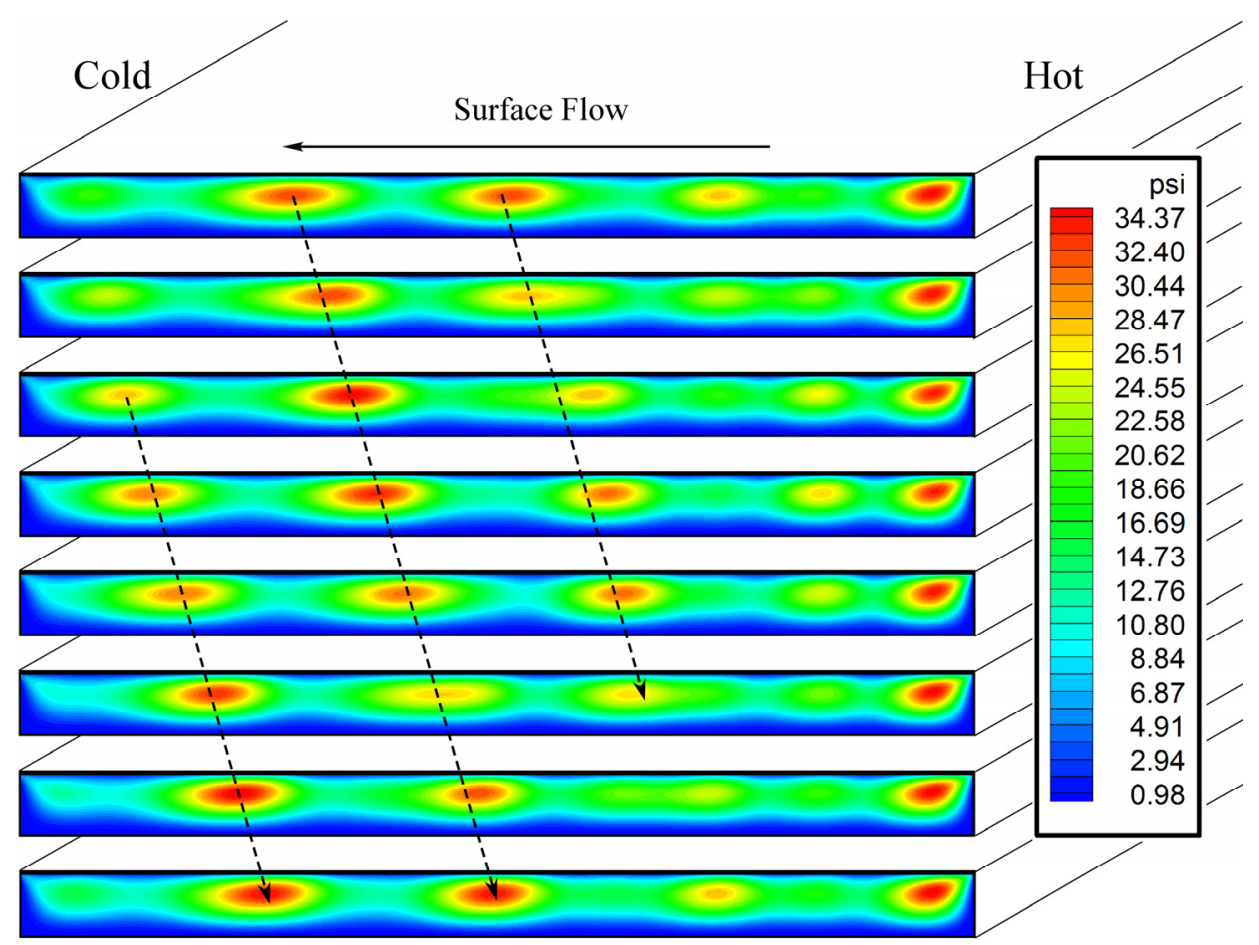

Figure 7: Travelling wave in a Newtonian fluid (eight snapshots evenly distributed during one period of oscillation, $\mathrm{Pr}=10, \mathrm{Ma}=2 \times 10^{4}$, layer with length-to-depth ratio $\mathrm{A}=15$, Ma defined as $\sigma_{\mathrm{T}} \Delta T \mathrm{~d} / \mu \alpha$ where $\mathrm{d}$ is the depth of the layer, adiabatic free surface; cold side on the left, hot side on the right; angular frequency of the wave $\omega \cong 38.9$ ). 
As illustrated in Fig. 7, the resulting effect is that of a chain or train of rolls, which are progressively displaced in time. In geometries with finite extent (shallow cavities) the number of visible rolls can be considered more or less constant in time since when a roll dies near the hot side, a new roll nucleates at the opposite end of the system (i.e. in proximity to the cold wall).

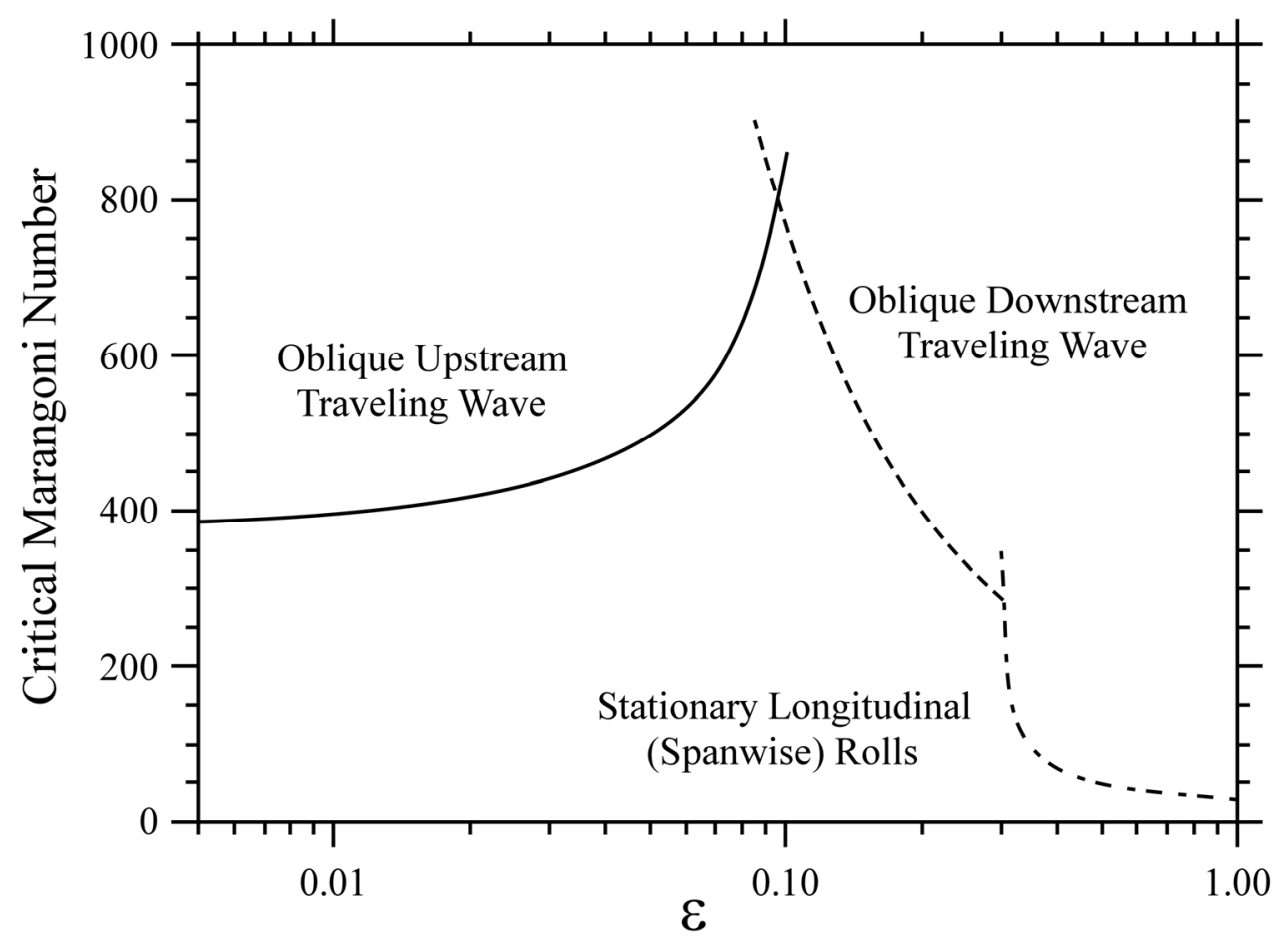

Figure 8: Critical Marangoni number as a function of the elasticity parameter (layer limited from above by an adiabatic free surface and from below by a adiabatic solid wall, temperature difference parallel to the interface, Marangoni number defined as $\mathrm{Ma}=\beta \sigma_{\mathrm{T}} \gamma \mathrm{d}^{2} / \mu \alpha$ where $\gamma$ is the uniform rate of temperature increase along the free surface, $\varepsilon \cong \operatorname{Pr}_{\mathrm{g}} \theta$ ) for $\operatorname{Pr}_{\mathrm{g}}=100$ and $\beta=0.1$ (reprinted from $\mathrm{Hu}$ et al., 2016, with the permission of AIP Publishing).

Though several numerical analyses have been appearing in the literature about these dynamics in Newtonian fluids (relevant examples along these lines being the two-dimensional computations by Peltier and Biringen (1993), Xu and Zebib (1998), Tang and $\mathrm{Wu}$ (2005), to the best of our knowledge there is only one (LSA) study where the instability of the return-flow solution has been considered in the presence of elastic effects.

Very recently, $\mathrm{Hu}$ et al. (2016) have found that on increasing the elasticity level of the fluid, the classical phenomena originally discovered by Smith and Davis (1983) (with waves that travel in the upstream direction) are taken over by a new mechanism where the waves change their sense of propagation (Fig. 8). More specifically, as soon as a given elasticity threshold is exceeded, the almost two-dimensional rolls described before, start to travel in the downstream direction, i.e. from the hot side towards the cold one (Fig. 9a). A second limit also exists in terms of elasticity such that if it is exceeded, waves (and related manifestations in the form of train of rolls) are completely suppressed and replaced by a steady convective pattern where the orientation of rolls becomes 
essentially longitudinal (i.e. the rolls extend along the spanwise direction with axes parallel to the temperature difference and they occupy fixed positions, i.e. the flow is stationary, Fig. 9b).
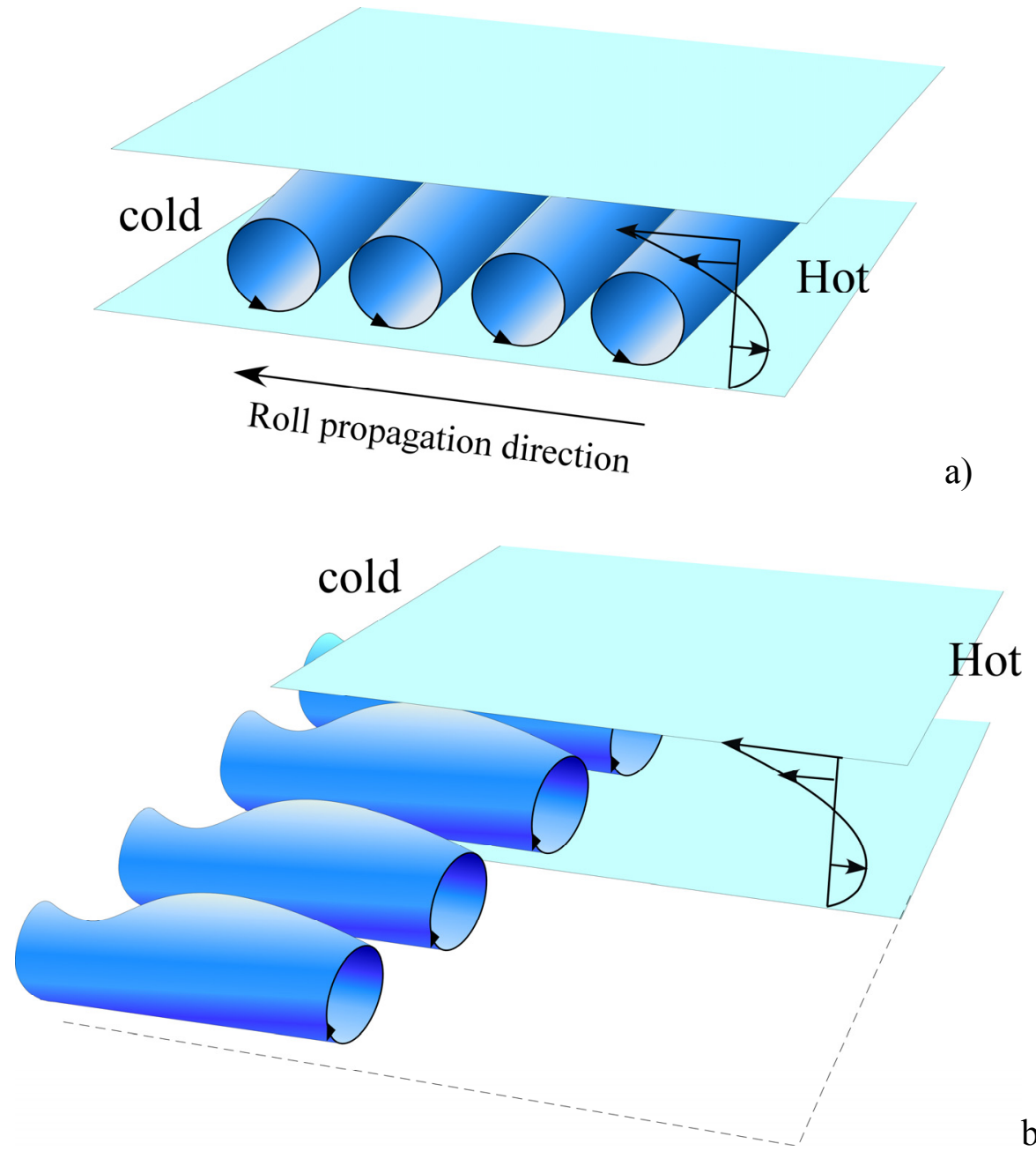

a)

b)

Figure 9: Sketches of the new convective modes made possible by elastic effects: a) Rolls traveling in the downstream direction, b) Stationary longitudinal rolls.

\section{Inertial and Viscoelastic Turbulence}

Having completed a description of the main types of thermal convection of natural origin and the fundamental instabilities which start to affect their behavior when relevant characteristic numbers are varied, in this final section we turn to examine the ultimate states of these flows, i.e. their behavior in the so-called turbulent regime. This condition is generally attained as the result of a sequence of instabilities, which bring the considered fluid system through a series of stages of evolution with increasing number of degrees of freedom until a very complex frequency spectrum is produced.

Such a discussion is not straightforward as one would imagine. There is some general consensus in the existing literature that the natural types of convection considered in this Chapter tend to follow completely different routes depending on whether elastic effects are present or not. This result might indeed have been anticipated on the basis of the notable differences that can be noticed 
already in regard to the first bifurcation of these flows (as illustrated in the preceding sections, e.g., the overstability effect for RB and MB convection discussed in Sects. 6 and 7, respectively).

Beyond the first bifurcation, for Newtonian liquids, the mechanisms of evolution towards chaos are essentially controlled by an increase in the Rayleigh and Marangoni numbers (for gravitational and surface-tension driven flows respectively). As these numbers are increased, new bifurcations occur, each contributing to enrich the initial flow with one or more new degrees of freedom. However, an equivalent behavior in viscoelastic fluids can be obtained even if $R a$ and/or Ma are kept constant by increasing the elasticity parameter.

In practical laboratory experiments, $R a$ or $M a$ can be made higher by applying a larger temperature difference to the considered liquid (this is equivalent to making the driving force progressively more important in comparison to the viscous forces that oppose fluid motion). Vice versa, a rise in $\theta$ can generally be obtained by increasing the viscosity of the polymer. A larger viscosity makes the relaxation time $\lambda$ (and therefore $\theta$ ) higher (as illustrated in Sect.2, $\lambda$ and $\eta$ are linearly proportional).

These apparently innocuous observations become particularly meaningful if one considers that they are equivalent to stating that for viscoelastic fluids flow instabilities can be excited using more viscous solutions. This is a relatively counter-intuitive concept with respect to the arguments generally used to explain turbulence in standard Newtonian fluids (Groisman and Steinberg, 2000).

An exhaustive review of the higher order bifurcations of thermogravitational and surface-tension driven flows and related hierarchy in standard Newtonian fluids can be found in the books by Lappa (2009 and 2012). Unfortunately, specific results relating to the equivalent sequence of instabilities for viscoelastic liquids are missing in the literature.

This implies that, in order to provide additional insights into these subjects purely heuristic arguments must necessarily be considered. Though the effective details of the progression towards chaos are lost in this way, this modus operandi is however effective in revealing the fundamental differences that have led over the years to consider a clear separation between two important (concurrent) concepts in fluid-dynamics, i.e. those of inertial and elastic turbulence.

Using widespread observational and phenomenological arguments, turbulence in standard Newtonian fluids is generally considered the consequence of an imbalance in the inertia of the fluid and the viscous forces. The relative importance of these two forces can be classically measured using the Reynolds number (typically defined as $\mathrm{Re}=\mathrm{V}_{\mathrm{REF}} \mathrm{L} / \mathrm{v}$ where $\mathrm{V}_{\mathrm{REF}}$ and $\mathrm{L}$ are a characteristic velocity and characteristic length, respectively, and $v$ is the kinematic viscosity of the fluid). When the Reynolds number becomes sufficiently high, it is assumed that inertia is able to overcome the counteracting effects of viscosity, which would otherwise tend to dissipate energy and maintain the flow stable (laminar).

This interpretation is actually at the root of some theories derived accordingly, which have proven successful in describing some important aspects of turbulence in common (Newtonian) fluids. A relevant example, is indeed, the theory of isotropic homogeneous turbulence (Kolmogorov, $1941 \mathrm{abc}, 1942)$. According to this model, when a turbulent equilibrium state is finally entered, the 
rate of energy dissipation due to frictional effects and the rate of energy injection (due to the considered driving force) become equal. In particular, by denoting by $\varepsilon$ this common rate, according to this theory energy is transferred at this (constant) rate from large scales (where energy is injected into the fluid) to shorter scales without undergoing significant dissipation (until a scale is reached where it is finally dissipated). This means that an intermediate range of scales can be postulated to exist where the energy spectrum is insensitive to the specific mechanisms of energy input and loss. Under a slightly different perspective, this specific behavior may be interpreted as the ability of the inertial flow to lose its memory in the chaotic scale-reduction mechanism depicted above, i.e. to develop in a certain range of scales (known as the inertial subrange) a universal behavior (which is at the root of the so-called similarity hypothesis, Kraichnan, 1974).

Though these concepts can effectively be applied to many circumstances when Newtonian fluids are considered, including the case of thermogravitational flows (the reader being referred, e.g., to Lappa and Gradisncak, 2018 and Lappa, 2019b for a rich set of examples), unfortunately, they are not applicable to elastic turbulence (see, e.g., Larson et al., 1990; Larson, 1992; Groisman and Steinberg, 1998; Morozov and van Saarloos, 2005).

Unlike inertial turbulence, elastic turbulence is not governed by the Reynolds number or equivalent parameters used to characterize other forms of convection (the Rayleigh and Marangoni numbers). Rather, as explained before, its development or onset must formally be put in direct relation with the elasticity number $(\theta)$.

Nevertheless, a relevant heuristic interpretation is still possible. In particular, meaningful arguments along these lines can be elaborated in their simplest form starting directly from the mathematical aspects covered in Sect. 2. As implicit in eq. (17), elastic stresses can evolve in time following an independent process with respect to the standard viscous (frictional) stresses. As an example, if the fluid were suddenly put in quiescent conditions (for such a case the viscous stresses would immediately disappear), the elastic counterpart would not vanish (these stresses would rather decay with the relaxation characteristic time).

This simple concept can be used to build an interesting explanation for the origin of elastic turbulence, which relies directly on the ability of these fluids to retain stresses even in the absence of a gradient of velocity. It can be argued that if an initial flow produces stretching of the long-chain molecules present in the liquid, the deformation of such molecules (evolving with a characteristic time that does not match that of the main flow) may cause secondary flows able to stretch them even further.

This fundamental mechanism may be seen as an internal feedback loop able to amplify an initial small disturbance through an iterative cause-and-effect coupling process that does not depend directly on the external forces driving the flow. As inertia is not directly involved, such a process is generally used to explain how chaos can be excited in these liquids using more viscous solutions. The feedback loops relying on the ability of molecules to be stretched and exert a back influence on the flow can excite new degrees of freedom and promote the development of turbulence (Groisman and Steinberg, 1998, 2000). 


\section{Conclusions}

In this chapter we have presented a focused review of the state-of-the-art concerning the dynamics of viscoelastic fluids when they are subjected to thermal stimuli in the presence of gravity (leading to buoyancy effects) and/or the liquid is directly in contact with air (i.e. a free interface exists that can support surface tension gradients). A key aim was to present a set of predictive links between the considered conditions (properties of the fluid and applied temperature difference) and the emerging behaviors (in terms of patterns and steady or time-dependent nature of the flow). Such a knowledge may help professionals and engineers working with these fluids to exert a better control on the related dynamics during process operations.

As reflected by the literature cited in the present chapter, finding numerical solutions to the NavierStokes equations in the presence of elasticity is extremely challenging and, indeed, only a handful of solutions have been appearing. Starting from the realization that the overwhelming majority of research on these subjects has focused on Newtonian fluids, we have exploited this observation to our advantage in order to develop a step-by-step treatment of the main modifications that are induced in the flow behavior when the level of elasticity is progressively increased.

These changes can be extremely counter-intuitive. As an example, while the concept of 'overstability' may be regarded as the relevant ingredient to characterize the peculiar dynamics of fluids uniformly heated from below ( $\mathrm{RB}$ and $\mathrm{MB}$ convection), it ceases to be valid when the direction of the temperature difference is rotated by 90 degrees (the trend becomes diametrically opposite). More precisely, in the former case, elasticity can make oscillatory solutions the most critical mode of convection (primary flow); by contrast, in the latter it can cause the replacement of time-dependent fluid motion with stationary rolls.

Similar considerations also apply to the ultimate state of these forms of convection. An increase in the viscosity, which for standard Newtonian fluids would produce stabilizing effects, can excite chaos in viscoelastic liquids, which explains why over the years a 'dichotomy' has been invoked to characterize properly the possible types of ("inertial' or "elastic") turbulence in these fluids.

As a concluding remark for this chapter, we wish to emphasize that the study of the path taken by thermally driven viscoelastic flows in their evolution towards chaos (the effective sequence of bifurcations that leads the system from the primary flow to the ultimate turbulent state) should be regarded as a subject still in early stages of development. It will certainly require additional efforts in the future in conjunction with the development of new (more realistic) mathematical models and more sophisticated simulations strategies. Hopefully, these new approaches will be able to overcome most of the well-known bottlenecks associated with the numerical analysis of these flows. The reader specifically interested in these technical challenges and the related concept of viscoelastic 'singularity' may consult, e.g., the theoretical studies by Renardy (1999), Demir et al. (1999), Owens and Phillips (2002), Bonito et al. (2011) and Siginer (2014). 


\section{References}

[-] Alves M.A., Oliveira P.J., Pinho F.T., (2003), "Benchmark solutions for the flow of Oldroyd-B and PTT fluids in planar contractions", J. Non-Newtonian Fluid Mech., 110, 45-75.

[-] Aly A.A., (2015), Heat Treatment of Polymers: A Review, International Journal of Materials Chemistry and Physics, 1(2), 132-140.

[-] Arshad T.A., Kim C.B., Prisco N.A., Katzenstein J.M., Janes D.W., Bonnecaze R.T. and Ellison C.J., (2014), Precision Marangoni-driven patterning, Soft Matter 10 (40), 8043-8050.

[-] Bénard H., (1900), "Les tourbillons cellulaires dans une nappe liquide", Rev. Gèn. Sci. Pure Appl., 11:1261-1271, 1309-1328

[-] Bénard H., (1901), "Les tourbillons cellulaires dans une nappe liquide transportant de la chaleur par convection en regime permanent", Annales de Chimie et de Physique, 23, 62-144.

[-] Bestehorn M., (1996), Square patterns in Bénard-Marangoni convection, Phys. Rev. Lett., 76,4649

[-] Bird R.B., Curtiss C.F., Armstrong R.C. and Hassager O., (1987), Dynamics of Polymeric Liquids, vol. 2, 2nd edn. John Wiley \& Sons, New York.

[-] Block M.J., (1956), "Surface tension as the cause of Bénard cells and surface deformation of a liquid film", Nature, 178, 650-651.

[-] Bonito A., Clément P., Picasso M., (2011), Viscoelastic Flows with Complex Free Surfaces: Numerical Analysis and Simulation, pp. 305-370 In Handbook of numerical analysis, Special Volume (Vol. XVI): Numerical Methods for Non-Newtonian Fluids, Guest Editors: R. Glowinski and J. Xu, Nort-Holland (Elsevier, 2011).

[-] Boussinesq J., (1903), "Théorie analytique de la chaleur, mise en harmonie avec la thermodynamique et avec la théorie mécanique de la lumière", Vol. 2, Paris, Gauthier-Villars, 1901-1903.

[-] Chan I. Chung, Extrusion of Polymers Theory \& Practice 2nd Edition, Hanser Publishers, Munich, Hanser Publications, Cincinnati.

[-] Colinet P., Legros J.C., Velarde M.G., (2001), Nonlinear Dynamics of Surface-Tension-Driven Instabilities, John Wiley, 2001

[-] Dauby P.C., Parmentier P., Lebon G., Grmela M., (1993), Coupled buoyancy and thermocapillary convection in a viscoelastic Maxwell fluid, J. Phys. Cond. Matter 5, 4343-4352.

[-] Dawson P.C., (1999), Flow Properties of Molten Polymers. In: Swallowe G.M. (eds) Mechanical Properties and Testing of Polymers. Polymer Science and Technology Series, vol 3. Springer, Dordrecht

[-] Dee G. T. and Sauer B. B., (1998), “The surface tension of polymer liquids", Adv. Phys. 47(2), 161-205.

[-] Demir H., Williams R.W. and Akyildiz, (1999), The singularities near the corner of a viscoelastic fluid in a 2D cavity, Mathematical and Computational Applications, 4(1), 39-44.

[-] Eltayeb, I. A., (1977), "Nonlinear thermal convection in an elastico-viscous layer heated from below", Proc. R. Soc. Lond. A 356, 161-176.

[-] Getachew D. and Rosenblat S., (1985), Thermocapillary instability of a viscoelastic liquid layer, Acta Mech. 85, 137-149.

[-] Giesekus H., (1966), “Die Elastizität von Flüssigkeiten”, Rheol. Acta, 5, 29-35.

[-] Grad, H., (1963), “Asymptotic Theory of the Boltzmann Equation”, Phys. Fluids, 6, 147-182.

[-] Green, T. (1968), "Oscillating convection in an elasticoviscous liquid", Phys. Fluids, 11, 14101412 .

[-] Grewell D. and Benatar A., (2007), "Welding of plastics: Fundamentals and new developments", Int. Polym. Process. 22(1), 43-60.

[-] Groisman A. and Steinberg V., (1998), "Mechanism of elastic instability in Couette flow of polymer solutions, Experiment," Phys Fluids, 10(10), 2451-2463. 
[-] Groisman A. and Steinberg V., (2000), "Elastic turbulence in a polymer solution flow," Nature, 405, 53-55.doi:10.1038/35011019.

[-] Guto E. B., Cohen E. D., and Kheboian G. I., (1995), Coating and Drying Defects (Wiley, New York, 1995).

[-] Hernández Hernández I.J. and Dávalos-Orozco L.A., (2015), Competition between stationary and oscillatory viscoelastic thermocapillary convection of a film coating a thick wall, International J. Therm. Sci., 89, 164-173.

[-] Hitt D. L. and Smith M. K., (1993), "Radiation-driven thermocapillary flows in optically thick liquid films", Phys. Fluids A 5(11), 2624-2632.

[-] Hu K.X., He M., and Chen Q.S, (2016), Instability of thermocapillary liquid layers for OldroydB fluid, Phys. Fluids, 28, 033105 (21 pages).

[-] Kolkka R.W. and Ierley G.R. (1987), On the convected linear stability of a viscoelastic Oldroyd B fluid heated form below, Journal of Non-Newtoman Fluid Mechanics, 25, 209-237

[-] Kolmogorov A.N., (1941a) "The local structure of turbulence in incompressible viscous fluids at very large Reynolds numbers", Dokl. Akad. Nauk. SSSR 30: 299-303. Reprinted in Proc. R. Soc. London A 434, 9-13 (1991).

[-] Kolmogorov A.N., (1941b), "On the degeneration of isotropic turbulence in an incompressible viscous fluids", Dokl. Akad. Nauk. SSSR 31, 538-541.

[-] Kolmogorov A.N., (1941c), "Dissipation of energy in isotropic turbulence", Dokl. Akad. Nauk. SSSR, 32, 19-21.

[-] Kolmogorov A.N., (1942) "Equations of turbulent motion in an incompressible fluid", Izv. Akad. Nauk. SSSR ser. Fiz. 6, 56-58.

[-] Kovalevskaya K.V. and Lyubimova T. P., (2011), "Onset and nonlinear regimes of convection of an elastoviscous fluid in a closed cavity heated from below", Fluid Dyn. 46, 854-863

[-] Kraichnan R.H., (1974), “On Kolmogorov's inertial-range theories”, J. Fluid Mech., 62, 305330 .

[-] Kramers H.A., (1944), "Het gedrag van macromoleculen in eenstroomendevloeistof", Physica, 11, 1-19.

[-] Krapivina E.N. and Lyubimova T. P., (2000), "Nonlinear regimes of convection of an elasticoviscous fluid in a closed cavity heated from below", Fluid Dyn., 35, 473-478.

[-] Lappa M. and Boaro, (2020), "Rayleigh-Bénard convection in viscoelastic liquid bridges", Under Review

[-] Lappa M., (2009), Thermal Convection: Patterns, Evolution and Stability, John Wiley \& Sons, Ltd (2009, Chichester, England).

[-] Lappa M., (2012), Rotating Thermal Flows in Natural and Industrial Processes, John Wiley \& Sons, Ltd (2012, Chichester, England).

[-] Lappa M., (2019a), On the Nature of Fluid-dynamics, Chapter 1 (pp. 1-64) in Understanding the Nature of Science, Patrick Lindholm Editor, Nova Science Publishers Inc., Series: Science, Evolution and Creationism, BISAC: SCI034000, ISBN: 978-1-53616-016-1. https://novapublishers.com/shop/understanding-the-nature-of-science/

[-] Lappa M., (2019b), "On the Highly Unsteady Dynamics of Multiple Thermal Buoyant Jets in Cross Flows”, Physics of Fluids, 31, 115105 (27 pages).

[-] Lappa M., and Ferialdi H., (2018), "Multiple solutions, Oscillons and Strange Attractors in ThermoViscoElastic Marangoni Convection”, Physics of Fluids, 30(10), 104104 (19 pages).

[-] Lappa M., and Gradinscak T., (2018), "On the Oscillatory Modes of Compressible Thermal Convection in inclined differentially heated cavities", Int. J. of Heat and Mass Transfer, 121, 412436.

[-] Larson R.G., Shaqfeh E.S.G., and Muller S.J., (1990), "A purely elastic instability in TaylorCouette flow," J. Fluid Mech., 218, 573-600.

[-] Larson, R. G. (1992), "Instabilities in viscoelastic flows", Rheol. Acta 31, 213-263. 
[-] Lebon G. and Cloot A., (1988), "An extended thermodynamic approach of non-Newtonian fluids and related results in Marangoni instability problem", J. Non-Newt. Fluid Mech., 28, 61-76.

[-] Lebon G., Parmentier P., Teller O., Dauby P.C., (1994), "Bénard-Marangoni instability in a viscoelastic Jeffreys' fluid layer", Rheol. Acta 33, 257-266.

[-] Li Z. and Khayat R.E., (2005), "Finite-amplitude Rayleigh-Bénard convection and pattern selection for viscoelastic fluids", J. Fluid Mech., 529, 221-251.

[-] Lyubimov D.V., Kovalevskaya K.V. and Lyubimova T.P., (2011), "Bifurcations in the problem of thermal convection of viscoelastic fluid in a closed cavity with free boundaries heated from below", Appl. Nonlinear Dyn. 19, 16-25.

[-] Lyubimov D.V., Kovalevskaya KV. and Lyubimova T.P., (2012), "Bifurcation analysis of a viscoelastic fluid heated from below”, Commun. Nonlinear Sci. Numer. Simul., 17, 3521-3532.

[-] Martínez-Mardones J. and Pérez-Garcí1a C., (1990), "Linear instability in viscoelastic fluid convection". J. Phys.: Condens. Matter 2, 1281-1290.

[-] Martínez-Mardones, J. and Pérez-Garcíia, C., (1992), "Bifurcation analysis and amplitude equations for viscoelastic convective fluids", Il Nuovo Cimento 14(9), 961-975.

[-] Martínez-Mardones, J., Tienmann, R., Walgraef, D. and Zeller, W., (1996), "Amplitude equations and pattern selection in viscoelastic convection", Phys. Rev. E 54, 1478-1488.

[-] Morozov A.N. and van Saarloos W., (2005), "Subcritical Finite-Amplitude Solutions for Plane Couette Flow of Viscoelastic Fluids," Phys. Rev. Lett., 95, 024501.

[-] Napolitano L.G., (1979), "Thermodynamics and dynamics of surface phases", Acta Astronaut., 6 (9), 1093-1112.

[-] Oberbeck A., (1879), "Ueber die Wärmeleitung der Flüssigkeiten bei Berücksichtigung der Strömungen infolge von Temperaturdifferenzen", Ann. Phys. Chem., 7, 271-292.

[-] Owens R. G. and Phillips T. N., (2002), Computational Rheology, Imperial College Press, 2002, London.

[-] Park H.M. and Lim J.Y., (2010), "A new numerical algorithm for viscoelastic fluid flow: the grid by-grid inversion method", J. Non-Newtonian Fluid Mech. 165, 238-246.

[-] Park H.M. and Park K.S., (2004), "Rayleigh-Bénard convection of viscoelastic fluids in arbitrary finite domains", Int. J. Heat Mass Transfer 47, 2251-2259

[-] Park H.M. and Ryu D.H., (2001b), "Hopf bifurcation in thermal convection of viscoelastic fluids within finite domains", J. Non-Newtonian Fluid Mech., 101, 1-19.

[-] Park H.M. and Ryu D.H., (2002), "Nonlinear convective stability problems of viscoelastic fluids in finite domains", Rheol. Acta 41, 427-440.

[-] Park H.M. and Ryu H.M., (2001a), "Rayleigh-Bénard convection of viscoelastic fluids in finite domains", J. Non-Newtonian Fluid Mech. 98, 169-184.

[-] Park H.M., (2018), "Peculiarity in the Rayleigh-Bénard convection of viscoelastic fluids", Int. J.Therm. Sci., 132, 34-41.

[-] Park H.M., Shin K.S., Sohn H.S., (2009), "Numerical simulation of thermal convection of viscoelastic fluids using the grid-by-grid inversion method", Int. J. Heat Mass Tran., 52, 48514861.

[-] Park, H. M. and Lee, H. S. (1996), "Hopf bifurcations of viscoelastic fluids heated from below", J. Non-Newtonian Fluid Mech. 66, 1-34.

[-] Parmentier, P., Lebon, G. and Regnier, V., (2000), "Weakly nonlinear analysis of BénardMarangoni instability in viscoelastic fluids", J. Non-Newtonian Fluid Mech., 89, 63-95.

[-] Pearson, J.R.A., (1958), "On convection cells induced by surface tension", J. Fluid Mech., 4, 489-500.

[-] Peltier L. and Biringen S., (1993), "Time-dependent thermocapillary convection in a rectangular cavity: numerical results for a moderate Prandtl number fluid", J. Fluid Mech., 257, 339-357.

[-] Ramkissoon H., Ramdath G., Comissiong D., and Rahaman K., (2006), “On thermal instabilities in a viscoelastic fluid", Int. J. Non-Linear Mech. 41(1), 18-25.

[-] Rauwendaal C., (2013), Polymer Extrusion, 5th Edition, 2013, Elsevier Science, 
[-] Rayleigh L., (1880), "On the stability or instability of certain fluid motions", Proc. Lond. Maths. Soc., 11, 57-70.

[-] Rayleigh L., (1916), "On the convection currents in a horizontal layer of fluid when the higher temperature is on the under side", Phil. Mag., 32, 529-546.

[-] Renardy M. (1999), High Weissenberg Number Asymptotics and Corner Singularities in Viscoelastic Flows. In: Durban D., Pearson J.R.A. (eds) IUTAM Symposium on Non-linear Singularities in Deformation and Flow. Springer, Dordrecht

[-] Revuz D. and Yor M., (1994), Continuous martingales and Brownian motion.Grundlehren der MathematischenWissenschaften [Fundamental Principles of Mathematical Sciences] Volume 293 (Springer-Verlag, Berlin).

[-] Rosenau, P., (1989), "Extending hydrodynamics via the regularization of the Chapman-Enskog expansion", Phys. Rev. A, 40, 7193-7196.

[-] Rosenblat S., (1986), "Thermal convection of a viscoelastic fluid", J. Non-Newtonian Fluid Mech. 21, 201-223.

[-] Rotheiser J., Joining of Plastics (Hanser, 1999).

[-] Saengow C., Giacomin A.J., Kolitawong C., (2015), "Extruding plastic pipe from eccentric dies", Journal of Non-Newtonian Fluid Mechanics 223, 176-199.

[-] Siddheshwar P.G., Sekhar G.N., Jayalatha G., (2011), "Surface tension driven convection in viscoelastic liquids with thermorheological effect", International Communications in Heat and Mass Transfer, 38, 468-473.

[-] Siginer D., 2014), Stability of Non-Linear Constitutive Formulations for Viscoelastic Fluids, Springer Briefs in Thermal Engineering and Applied Science (2014 , Springer International Publishing, New York).

[-] Smith M.K., Davis S.H. (1983), "Instabilities of dynamic thermocapillary liquid layers. Part 1 : convective instabilities ", J. Fluid Mech, 132, 119-144.

[-] Sokolov, M. and Tanner, R. I. (1972), "Convective stability of a general viscoelastic fluid heated from below", Phys. Fluids 15, 534-539.

[-] Tang Z.M. and Hu W.R., (2005), "Hydrothermal Wave in a Shallow Liquid Layer", Microgravity Sci. Tech., 16(1), 253-258.

[-] Troughton M. J., Handbook of Plastics Joining: A Practical Guide (William Andrew, 2008).

[-] Vest, C. M. and Arpaci, V. S., (1969), "Overstability of a viscoelastic fluid layer heated from below", J. Fluid Mech. 36, 613-623.

[-] Wilson S.R.D., (1995), Growth rates of the Marangoni instability in a layer of elastic fluid, Rheol. Acta, 34, 601-605.

[-] Xu J. and Zebib A., (1998), "Oscillatory two- and three-dimensional thermocapillary convection", J. Fluid Mech., 364, 187-209. 